\begin{tabular}{|c|l|}
\hline Title & Singularities of hy perbolic Gauss maps \\
\hline Author(s) & Izumiya, S.; Pei, D-H; Sano, T. \\
\hline Citation & Hokkaido University Preprint Series in Mathematics, 514, 1-27 \\
\hline Issue Date & 2001-1-1 \\
\hline DOI & 10.14943/83660 \\
\hline Doc URL & http://hdl.handle.net/2115/69264 \\
\hline Type & bulletin (article) \\
\hline File Information & pre514.pdf \\
\hline
\end{tabular}

Instructions for use 


\section{Singularities of hyperbolic Gauss maps}

Shyuichi IZUMIYA, Dong-he PEI and Takasi SANO

Series \#514. January 2001 


\section{HOKKAIDO UNIVERSITY PREPRINT SERIES IN MATHEMATICS}

\#486 T. Nakazi, Functions in $N_{+}$with the positive real parts on the boundary, 21 pages. 2000.

\#487 Y. Shibukawa, Classification of the $R$-operator, 36 pages. 2000.

\#488 A. Inoue, Asymptotic behaviour for partial autocorrelation functions of fractional ARIMA processes, 20 pages. 2000.

\#489 S. Ohtani, Construction of unramified Galois extensions over maximal abelian extensions of algebraic number fields, 14 pages. 2000.

\#490 T. Nakazi and T. Yamamoto, The real part of an outer function and a Helson-Szegö weight, 13 pages. 2000.

\#491 A. Yamagami, On Gouvêás conjecture on controlling the conductor, 11 pages. 2000.

\#492 I. Tsuda and M. Hatakeyama, Making sense of internal logic: Theory and a case study, 10 pages. 2000.

\#493 I. Tsuda, Towards an interpretation of dynamic neural activity in terms of chaotic dynamical systems, 73 pages. 2000.

\#494 T. Mikami, Optimal control for absolutely continuous stochastic processes and the mass transportation problem, 17 pages. 2000 .

\#495 M. Arisawa and Y. Giga, Anisotropic curvature flow in a very thin domain, 21 pages. 2000.

\#496 T. Nakazi, Backward shift invariant subspaces in the bidisc, 9 pages. 2000.

\#497 Y. Giga, K. Inui, J. Kato and S. Matsui, Remarks on the uniqueness of bounded solutions of the NavierStokes equations, 4 pages. 2000.

\#498 Y. Giga, M. Paolini and P. Rybka, On the motion by singular interfacial energy, 21 pages. 2000.

\#499 J. Escher and Y. Giga, On a limiting motion and self-interactions of curves moved by the intermediate surface diffusion flow, 12 pages. 2000.

\#500 I. Tsuda and S. Kuroda, Cantor coding in the hippocampus, 20 pages. 2000.

\#501 M. Tsujii, Fat solenoidal attractors, 20 pages. 2000.

\#502 A. Arai, Ground state of the massless Nelson model without infrared cutoff in a non-Fock representation, 19 pages. 2000.

\#503 Y. Giga, S. Matsui and O. Sawada, Global existence of two-dimensional Navier-Stokes flow with nondecaying initial velocity, 19 pages. 2000.

\#504 A. Inoue and Y. Kasahara, Partial autocorrelation functions of the fractional ARIMA processes with negative degree of differencing, 14 pages. 2000.

\#505 T. Nakazi, Interpolation problem for $\ell^{1}$ and a uniform algebra, 12 pages. 2000.

\#506 R. Kobayashi and Y. Giga, On anisotropy and curvature effects for growing crystals, 38 pages. 2000.

\#507 A. Arai, Instability in the spectral and the Fredholm properties of an infinite dimensional Dirac operator on the abstract Boson-Fermion Fock space, 6 pages. 2000.

\#508 A. Arai, Supersymmetric methods for constructing soliton-type solutions to multi-component nonlinear Schrödinger and Klein-Gordon equations, 22 pages. 2000.

\#509 Y. Tonegawa, Phase field model with a variable chemical poteritial, 30 pages. 2000.

\#510 Y. Giga, Shocks and very strong vertical diffusion, 11 pages. 2000. 


\title{
Singularities of hyperbolic Gauss maps
}

\author{
Shyuichi IZUMIYA \\ Department of Mathematics, Hokkaido University, \\ Sapporo 060-0810, Japan , \\ e-mail : izumiya@math.sci.hokudai.ac.jp \\ Dong-he PEI \\ Department of Mathematics, North East Normal University, \\ Chang Chun 130024, P.R.China \\ e-mail: northlcd@public.cc.jl.cn \\ and \\ Takasi SANO \\ Faculty of engineering, Hokkaigakuen University, \\ Sapporo 062-8605, Japan \\ e-mail : t-sano@cvl.hokkai-s-u.ac.jp
}

\begin{abstract}
In this paper we adopt the Hyperboloid in Minkowski space as the model of Hyperbolic space. We define the hyperbolic Gauss map and the hyperbolic Gauss indicatrix of a hypersurface in Hyperbolic space. The hyperbolic Gauss map has been introduced by Epstein[7] in the Poincaré ball model which is very useful for the study of constant mean curvature surfaces. However, it is very hard to proceed the calculation because it has an intrinsic form. Here, we give an extrinsic definition and we study singularities of these. In the study of singularities of the hyperbolic Gauss map (indicatrix), we understand that the hyperbolic Gauss indicatrix is much easier to proceed the calculation. We introduce the notion of hyperbolic Gauss-Kronecker curvature whose zero sets correspond to the singular set of the hyperbolic Gauss map (indicatrix). We also develop a local differential geometry of hypersurfaces concerning on contact with hyperhorospheres.
\end{abstract}

\section{Introduction}

In this paper we study extrinsic differential geometry on hypersurfaces in Hyperbolic space as applications of singularity theory. In [3] Bleeker and Wilson studied the singularities of the Gauss map of a surface in Euclidean 3-space. In their paper the main theorem asserts

1991 Mathematics Subject classification. 53A25, 53A05, 58C27

Keywords and Phrase. Hyperbolic space, hypersurfaces, hyperbolic Gauss maps, singularities 
that the generic singularities of Gauss maps are folds or cusps. Later that Banchoff et al [2],Landis[12] and Platonova[21] have studied geometric meanings of cusps of the Gauss map of a surface. Bruce[4] and Romero-Fuster[20] have also independently studied the singularities of the Gauss map and the dual of a hypersurface in Euclidean space. The singularity of the dual of a hypersurface is deeply related to the singularity of the Gauss map of the hypersurface. Their main tools for the study is the height function on a hypersurface. It has been classically known that the singular set of the Gauss map is the parabolic set of the surface and it can be interpreted as the criminant set of the height function. This is the reason why they adopted the height function for the study of Gauss maps. They applied the deformation theory of smooth functions to the height function and derived geometric results on the Gauss maps. We can interpret that these results on Gauss maps describe the contact between hypersurfaces and hyperplanes. It is called the "flat geometry" of hypersurfaces in Euclidean space. It also has been known that Gauss maps of hypersurfaces are Lagrangian maps. Moreover, the generic singularities of Gauss maps of hypersurfaces and Lagrangian maps are the same[1]. Singularities of projective Gauss maps are also studied by McCrory et al [16, 17]. There are many other articles concerning on singularities of Gauss maps, we only refer the reference of the book [2] here.

On the other hand, Epstein [7] introduced the notion of the hyperbolic Gauss map of a surface in the Poincaré ball model of Hyperbolic space. However, it is very hard to study the singularities of the hyperbolic Gauss map because it is given as an intrinsic expression. Here, we adopt the Hyperboloid in Minkowski space as the model of Hyperbolic space. Under this framework, we can explicitly express the hyperbolic Gauss map of a hypersurface by using the local parametrisation of the hypersurface. In $\S 2$ we introduce the notion of the lightcone normal, the hyperbolic Gauss map and the hyperbolic Gauss indicatrix of a hypersurface in Hyperbolic space. Readers can easily recognise that the hyperbolic Gauss indicatrix is much easier to study compared with the hyperbolic Gauss map. Corresponding to these notions, we define two hyperbolic invariants. One is the hyperbolic Gauss-Kronecker curvature and another is the hyperbolic mean curvature. We only consider the geometric meaning of the hyperbolic Gauss-Kronecker curvature here. Comparing with the ordinary Gauss-Kronecker curvature, the hyperbolic Gauss-Kronecker curvature is not an intrinsic invariant. It depends on the embedding of the hypersurface into Hyperbolic space. One of our conclusions asserts that the hyperbolic Gauss-Kronecker curvature is a local invariant which describe the contact between hypersurfaces and hyperhoroshperes. This means that we establish the "horospherical geometry" of hypersurfaces in Hyperbolic space. We do not know that these hyperbolic invariants are essentially new or not. This is the authors' future problem.

All maps considered here are of class $C^{\infty}$ unless otherwise stated.

Acknowledgments This article has been completed during the first author's stay at Isaac Newton institute for Mathematical Sciences, Cambridge university 2000 as a participant of the special semester of Singularity theory. The authors would like to thank all staffs of the institute and the organizers of the semester for their kind hospitality and perfect organisation.

\section{The extrinsic hyperbolic differential geometry}

In this section we develop the (local) hyperbolic differential geometry in the explicit way. Here, we adopt the Lorentzian model of the hyperbolic $n$-space.

Let $\mathbb{R}^{n+1}=\left\{\left(x_{0}, x_{1}, \ldots, x_{n}\right) \mid x_{i} \in \mathbb{R}(i=0,1, \ldots, n)\right\}$ be an $(n+1)$-dimensional vector 
space. For any $\boldsymbol{x}=\left(x_{0}, x_{1}, \ldots, x_{n}\right), \boldsymbol{y}=\left(y_{0}, y_{1}, \ldots, y_{n}\right) \in \mathbb{R}^{n+1}$, the pseudo scalar product of $\boldsymbol{x}$ and $\boldsymbol{y}$ is defined by

$$
\langle\boldsymbol{x}, \boldsymbol{y}\rangle=-x_{0} y_{0}+\sum_{i=1}^{n} x_{i} y_{i}
$$

We call $\left(\mathbb{R}^{n+1},\langle\rangle,\right)$ Minkowski $(n+1)$-space. We denote $\mathbb{R}_{1}^{n+1}$ instead of $\left(\mathbb{R}^{n+1},\langle\rangle,\right)$. We say that a non-zero vector $\boldsymbol{x} \in \mathbb{R}_{1}^{n+1}$ is spacelike, lightlike or timelike if $\langle\boldsymbol{x}, \boldsymbol{x}\rangle>0,\langle\boldsymbol{x}, \boldsymbol{x}\rangle=0$ or $\langle\boldsymbol{x}, \boldsymbol{x}\rangle<0$ respectively. For a vector $\boldsymbol{v} \in \mathbb{R}_{1}^{n+1}$ and a real number $c$, we define the hyperplane with pseudo normal $\boldsymbol{v}$ by

$$
H P(\boldsymbol{v}, c)=\left\{\boldsymbol{x} \in \mathbb{R}_{1}^{n+1} \mid\langle\boldsymbol{x}, \boldsymbol{v}\rangle=c\right\} .
$$

We call $H P(\boldsymbol{v}, c)$ a spacelike hyperplane, a timelike hyperplane or a lightlike hyperplane if $\boldsymbol{v}$ is timelike, spacelike or lightlike respectively.

We now define Hyperbolic $n$-space by

$$
H_{+}^{n}(-1)=\left\{\boldsymbol{x} \in \mathbb{R}_{1}^{n+1} \mid\langle\boldsymbol{x}, \boldsymbol{x}\rangle=-1, x_{0} \geq 1\right\}
$$

and de Sitter $n$-space by

$$
S_{1}^{n}=\left\{\boldsymbol{x} \in \mathbb{R}_{1}^{n+1} \mid\langle\boldsymbol{x}, \boldsymbol{x}\rangle=1\right\} .
$$

For any $\boldsymbol{x}_{1}, \boldsymbol{x}_{2}, \ldots, \boldsymbol{x}_{n} \in \mathbb{R}_{1}^{n+1}$, we define a vector $\boldsymbol{x}_{1} \wedge \boldsymbol{x}_{2} \wedge \cdots \wedge \boldsymbol{x}_{n}$ by

$$
\boldsymbol{x}_{1} \wedge \boldsymbol{x}_{2} \wedge \cdots \wedge \boldsymbol{x}_{n}=\left|\begin{array}{cccc}
-e_{0} & e_{1} & \cdots & \boldsymbol{e}_{n} \\
x_{0}^{1} & x_{1}^{1} & \cdots & x_{n}^{1} \\
x_{0}^{2} & x_{1}^{2} & \cdots & x_{n}^{2} \\
\vdots & \vdots & \cdots & \vdots \\
x_{0}^{n} & x_{1}^{n} & \cdots & x_{n}^{n}
\end{array}\right|
$$

where $\boldsymbol{e}_{0}, \boldsymbol{e}_{1}, \ldots, \boldsymbol{e}_{n}$ is the canonical basis of $\mathbb{R}_{1}^{n+1}$ and $\boldsymbol{x}_{i}=\left(x_{0}^{i}, x_{1}^{i}, \ldots, x_{n}^{i}\right)$. We can easily check that

$$
\left\langle\boldsymbol{x}, \boldsymbol{x}_{1} \wedge \boldsymbol{x}_{2} \wedge \cdots \wedge \boldsymbol{x}_{n}\right\rangle=\operatorname{det}\left(\boldsymbol{x}, \boldsymbol{x}_{1}, \ldots, \boldsymbol{x}_{n}\right),
$$

so that $\boldsymbol{x}_{1} \wedge \boldsymbol{x}_{2} \wedge \cdots \wedge \boldsymbol{x}_{n}$ is pseudo orthogonal to any $\boldsymbol{x}_{i}(i=1, \ldots, n)$.

We also define a set $L C_{a}=\left\{\boldsymbol{x} \in \mathbb{R}_{1}^{n+1} \mid\langle\boldsymbol{x}-\boldsymbol{a}, \boldsymbol{x}-\boldsymbol{a}\rangle=0\right\}$, which is called a closed lightcone with the vertex $\boldsymbol{a}$. We denote that

$$
L C_{+}^{*}=\left\{\boldsymbol{x}=\left(x_{0}, \ldots x_{n}\right) \in L C_{0} \mid x_{0}>0\right\}
$$

and we call it the future lightcone at the origin. We can also define the notion of the past lightcone. If $\boldsymbol{x}=\left(x_{0}, x_{1}, \ldots, x_{n}\right)$ is a lightlike vector, then $x_{0} \neq 0$. Therefore we have

$$
\tilde{\boldsymbol{x}}=\left(1, \frac{x_{1}}{x_{0}}, \ldots, \frac{x_{n}}{x_{0}}\right) \in S_{+}^{n-1}=\left\{\boldsymbol{x}=\left(x_{0}, x_{1}, \ldots, x_{n}\right) \mid\langle\boldsymbol{x}, \boldsymbol{x}\rangle=0, x_{0}=1\right\} .
$$

Here, we call $S_{+}^{n-1}$ the lightcone $(n-1)$-sphere.

We now construct the extrinsic differential geometry on hypersurfaces in $H_{+}^{n}(-1)$. Let

$$
\boldsymbol{x}: U \longrightarrow H_{+}^{n}(-1)
$$


be a regular hypersurface (i.e., an embedding), where $U \subset \mathbb{R}^{n-1}$ is an open subset. We denote that $M=\boldsymbol{x}(U)$ and identify $M$ and the embedding $\boldsymbol{x}$. Since $\langle\boldsymbol{x}, \boldsymbol{x}\rangle \equiv-1$, we have

$$
\left\langle\boldsymbol{x}_{u_{i}}, \boldsymbol{x}\right\rangle \equiv 0 \quad(i=1, \ldots, n-1),
$$

where $u=\left(u_{1}, \ldots u_{n-1}\right) \in U$. Define a vector

$$
\boldsymbol{e}(u)=\frac{\boldsymbol{x}(u) \wedge \boldsymbol{x}_{u_{1}}(u) \wedge \cdots \wedge \boldsymbol{x}_{u_{n-1}}(u)}{\left\|\boldsymbol{x}(u) \wedge \boldsymbol{x}_{u_{1}}(u) \wedge \cdots \wedge \boldsymbol{x}_{u_{n-1}}(u)\right\|},
$$

then we have

$$
\left\langle\boldsymbol{e}, \boldsymbol{x}_{u_{i}}\right\rangle \equiv\langle\boldsymbol{e}, \boldsymbol{x}\rangle \equiv 0, \quad\langle\boldsymbol{e}, \boldsymbol{e}\rangle \equiv 1 .
$$

Therefore the vector $\boldsymbol{x} \pm \boldsymbol{e}$ is lightlike. Since $\boldsymbol{x}(u) \in H_{+}^{n}(-1)$ and $\boldsymbol{e}(u) \in S_{1}^{n}$, we can show that $\boldsymbol{x}(u) \pm \boldsymbol{e}(u) \in L C_{+}^{*}$. We define a map

$$
\mathbb{L}^{ \pm}: U \longrightarrow L C_{+}^{*}
$$

by $\mathbb{L}^{ \pm}(u)=\boldsymbol{x}(u) \pm \boldsymbol{e}(u)$ which is called the hyperbolic Gauss indicatrix (or the lightcone dual) of $\boldsymbol{x}$. We also define a map

$$
\widetilde{\mathbb{L}^{ \pm}}: U \longrightarrow S_{+}^{n-1}
$$

by $\widetilde{\mathbb{L}^{ \pm}}(u)=\widetilde{\mathbb{L}^{ \pm}(u)}$ which is called the hyperbolic Gauss map of $\boldsymbol{x}$.

We remark that the notion of the hyperbolic Gauss map is defined in [7] for a surface in the Poincaré disk model and our definition is equivalent to the definition in [7] in the case when $n=3$.

On the other hand, we define a map $\mathbb{E}: U \longrightarrow S_{1}^{n}$ by $\mathbb{E}(u)=\boldsymbol{e}(u)$ which is called the ordinary Gauss indicatrix of $\boldsymbol{x}$.

We can construct an extrinsic differential geometry on $\boldsymbol{x}$ by using the unit normal $\boldsymbol{e}$ like as the unit normal of a hypersurface in Euclidean space. In this case, the ordinary Gauss indicatrix of a hypersurface plays the similar role like as the Gauss map of a hypersurface in Euclidean space plays. It is also an interesting project. We do not, however, study the properties of the ordinary Gauss indicatrix here.

In order to define the hyperbolic Gauss-Kronecker curvature and the hyperbolic mean curvature of the hypersurface $M=\boldsymbol{x}(U)$, we have the following fundamental lemma.

Lemma 2.1 For any $p=\boldsymbol{x}\left(u_{0}\right) \in M$ and $\boldsymbol{v} \in T_{p} M$, we have $D_{v} e \in T_{p} M$, so that $D_{v} \mathbb{L}^{ \pm} \in T_{p} M$. Here, $D_{v}$ denotes the covariant derivative with respect to the tangent vector $v$.

Proof. Since $\left\{\boldsymbol{x}, \boldsymbol{e}, \boldsymbol{x}_{u_{1}}, \ldots, \boldsymbol{x}_{u_{n-1}}\right\}$ is a basis of the vector space $T_{p} \mathbb{R}_{1}^{n+1}$, we have $D_{v} \boldsymbol{e}=$ $\lambda \boldsymbol{x}+\eta \boldsymbol{e}+\lambda_{1} \boldsymbol{x}_{u_{1}}+\cdots+\lambda_{n-1} \boldsymbol{x}_{u_{n-1}}$ for some real numbers $\lambda, \eta, \lambda_{1}, \ldots, \lambda_{n-1}$. It follows form the fact $\langle\boldsymbol{e}, \boldsymbol{e}\rangle=0$ that we have $\boldsymbol{v}(\langle\boldsymbol{e}, \boldsymbol{e}\rangle)=0$. On the other hand, we have $2\left\langle D_{v} \boldsymbol{e}, \boldsymbol{e}\right\rangle=\boldsymbol{v}(\langle\boldsymbol{e}, \boldsymbol{e}\rangle)=0$, so that $\eta=0$. It also follows from the fact $\langle\boldsymbol{e}, \boldsymbol{x}\rangle\left\langle\boldsymbol{e}, D_{v} \boldsymbol{x}\right\rangle=0$ that $\lambda=0$. Since $\boldsymbol{x}_{u_{1}}, \ldots, \boldsymbol{x}_{u_{n-1}}$ is the basis of $T_{p} M, D_{v} e \in T_{p} M$. By definition, we have $D_{v} \boldsymbol{x}=\mu_{1} \boldsymbol{x}_{u_{1}}+\cdots+\mu_{n-1} \boldsymbol{x}_{u_{n-1}}$ for some $\mu_{1}, \ldots, \mu_{n-1}$. Therefore, we have $D_{v} \mathbb{L}^{ \pm}=D_{v} x \pm D_{v} e \in T_{p} M$.

We now consider the geometric meaning of the hyperbolic Gauss map and the hyperbolic indicatrix of a hypersurface. The model hypersurface in Euclidean differential geometry is a hyperplane or a hypersphere. It might be said that singularities of the Gauss map (i.e., parabolic points) estimate how the hypersurface is different from (or, the same as) a hyperplane (i.e, so 
called, the "flat geometry"). Here, we consider the following model hypersurfaces in Hyperbolic space. A hypersurface given by the intersection of $H_{+}^{n}(-1)$ and a spacelike hyperplane, a timelike hyperplane or a lightlike hyperplane is respectively called a hypersphere, a equidistant hyperplane or a hyperhorosphere. Then we have the following proposition.

Proposition 2.2 Let $\boldsymbol{x}: U \longrightarrow H_{+}^{n}(-1)$ be a hypersurface in $H_{+}^{n}(-1)$. If the hyperbolic Gauss indicatrix $\mathbb{L}^{ \pm}$is constant, then the hypersurface $\boldsymbol{x}(U)$ is a part of a hyperhorosphere.

Proof. Since $\mathbb{L}^{ \pm}=\boldsymbol{x} \pm \boldsymbol{e}$ is constant, the set $V=\left\{\boldsymbol{y} \in \mathbb{R}_{1}^{n+1} \mid\langle\boldsymbol{y}, \boldsymbol{x} \pm \boldsymbol{e}\rangle=0\right\}$ is a lightlike hyperplane. For any $u, u^{\prime} \in U$, we now show that $V+\boldsymbol{x}(u)=V+\boldsymbol{x}\left(u^{\prime}\right)$. Since $(\boldsymbol{x} \pm \boldsymbol{e})(u)=(\boldsymbol{x} \pm \boldsymbol{e})\left(u^{\prime}\right)$, we have $\boldsymbol{x}(u)=\boldsymbol{x}\left(u^{\prime}\right) \pm\left(\boldsymbol{e}\left(u^{\prime}\right)-\boldsymbol{e}(u)\right)$. It is enough to show that $\boldsymbol{e}\left(u^{\prime}\right)-\boldsymbol{e}(u) \in V$. We can calculate that $\langle\boldsymbol{e}(u), \boldsymbol{x} \pm \boldsymbol{e}\rangle=\left\langle\boldsymbol{e}\left(u^{\prime}\right), \boldsymbol{x} \pm \boldsymbol{e}\right\rangle= \pm 1$, so that we have $\left\langle\boldsymbol{e}\left(u^{\prime}\right)-\boldsymbol{e}(u), \boldsymbol{x} \pm \boldsymbol{e}\right\rangle=0$. This means that $\boldsymbol{e}\left(u^{\prime}\right)-\boldsymbol{e}(u) \in V$.

Therefore, $V+\boldsymbol{x}(u)$ is a constant affine hyperplane in $\mathbb{R}_{1}^{n+1}$ and we have $\boldsymbol{x}(U) \subset V+\boldsymbol{x}(u) \cap$ $H_{+}^{n}(-1)$. Here, $V+\boldsymbol{x}(u) \cap H_{+}^{n}(-1)$ is a hyperhorosphere.

Since two pseudo-normals to a lightlike hyperplane are constant, the converse is true.

We remark that if the hyperbolic Gauss map $\widetilde{\mathbb{L}^{ \pm}}$is constant, then the hyperbolic Gauss indicatrix $\mathbb{L}^{ \pm}$is also constant, so that the surface is a part of a hyperhorosphere. In Euclidean differential geometry, if the Gauss map of a surface is constant, then the surface is a part of a hyperplane. Therefore, we now treat hyperhorospheres in our theory like as hyperplanes in Euclidean differential geometry. We would establish the "horospherical geometry" as an application of singularity theory in this paper.

Under the identification of $\boldsymbol{x}$ and $M$, the derivative $d \boldsymbol{x}\left(u_{0}\right)$ can be identified to the identity mapping $i d_{T_{p} M}$ on the tangent space $T_{p} M$, where $p=\boldsymbol{x}\left(u_{0}\right)$. This means that $d \mathbb{L}^{ \pm}\left(u_{0}\right)=i d_{T_{p} M} \pm$ $d e\left(u_{0}\right)$. By Lemma 2.1, de $\left(u_{0}\right)$ is also a linear transformation on the tangent space $T_{p} M$. We call the linear transformation $S_{p}^{ \pm}=-d \mathbb{L}\left(u_{0}\right): T_{p} M \longrightarrow T_{p} M$ the hyperbolic shape operator of $M=\boldsymbol{x}(U)$ at $p=\boldsymbol{x}\left(u_{0}\right)$. We also call the linear transformation $A_{p}=-\operatorname{de}\left(u_{0}\right): T_{p} M \longrightarrow T_{p} M$ the (ordinary) shape operator of $M=\boldsymbol{x}(U)$ at $p=\boldsymbol{x}\left(u_{0}\right)$. We denote the eigenvalue of $S_{p}^{ \pm}$by $\bar{\kappa}_{p}^{ \pm}$and the eigenvalue of $A_{p}$ by $\kappa_{p}$. By the relation $S_{p}^{ \pm}=-i d_{T_{p} M} \pm A_{p}, S_{p}^{ \pm}$and $A_{p}$ have same eigenvectors and we have a relation that $\bar{\kappa}_{p}^{ \pm}=-1 \pm \kappa_{p}$.

We now define the notion of hyperbolic curvatures as follows: The hyperbolic GaussKronecker curvature of $M=\boldsymbol{x}(U)$ at $p=\boldsymbol{x}\left(u_{0}\right)$ is defined to be

$$
K_{h}^{ \pm}\left(u_{0}\right)=\operatorname{det} S_{p}^{ \pm}
$$

The hyperbolic mean curvature of $M=\boldsymbol{x}(U)$ at $p=\boldsymbol{x}\left(u_{0}\right)$ is defined to be

$$
H_{h}^{ \pm}\left(u_{0}\right)=\frac{1}{n-1} \operatorname{Trace} S_{p}^{ \pm}
$$

Since $A_{p}$ is the shape operator with respect to the Riemannian metric on $M$ induced from the Lorentzian metric on $\mathbb{R}_{1}^{n}$, the (ordinary) Gauss-Kronecker curvature is $K\left(u_{0}\right)=\operatorname{det} A_{p}$ and the (ordinary) mean curvature is $H\left(u_{0}\right)=\frac{1}{n-1}$ Trace $A_{p}$. By definition, we have the relation $H_{h}^{ \pm}(u)= \pm H(u)-1$. In the theory of surfaces in Hyperbolic space with constant mean curvature, the surfaces with $H \equiv \pm 1$ are the most important class. These surfaces corresponds to surfaces with $H_{h}^{ \pm} \equiv 0$. These might be called hyperbolic minimal surfaces. However, we do not consider the hyperbolic mean curvature in this paper. 
We say that a point $u \in U$ or $p=\boldsymbol{x}(u)$ is an umbilic point if $S_{p}^{ \pm}=\bar{\kappa}^{ \pm}(p) i d_{T_{p} M}$. Since the eigenvectors of $S_{p}^{ \pm}$and $A_{p}$ are same, the above condition is equivalent to the condition $A_{p}= \pm \kappa(p) i d_{T_{p} M}$. We say that $M=\boldsymbol{x}(U)$ is totally umbilic if all points on $M$ are umbilic. In [6], Cecile and Ryan have characterized totally umbilic submanifolds by using three different functions on Hyperbolic space. Here, we have the following classification theorem:

Proposition 2.3 Suppose that $M=\boldsymbol{x}(U)$ is totally umbilic, then $\bar{\kappa}^{ \pm}(p)$ is constant $\bar{\kappa}^{ \pm}$. Under this condition, we have the following classification:

1) Suppose that $\bar{\kappa}^{ \pm} \neq 0$.

a) If $\bar{\kappa}^{ \pm} \neq-1$ and $\left|\bar{\kappa}^{ \pm}+1\right|<1$, then $M$ is a part of an equidistant hyperplane.

b) If $\bar{\kappa}^{ \pm} \neq-1$ and $\left|\bar{\kappa}^{ \pm}+1\right|>1$, then $M$ is a part of a hypersphere.

c) If $\bar{\kappa}^{ \pm}=-1$, then $M$ is a part of a hyperplane.

2) If $\bar{\kappa}^{ \pm}=0$, then $M$ is a part of a hyperhorosphere.

Proof. By definition, we have $-\mathbb{L}_{u_{i}}^{ \pm}=\bar{\kappa}^{ \pm} \boldsymbol{x}_{u_{i}}$ for $i=1, \ldots, n-1$. Therefore, we have

$$
-\mathbb{L}_{u_{i} u_{j}}^{ \pm}=\bar{\kappa}_{u_{j}}^{ \pm} \boldsymbol{x}_{u_{i}}+\bar{\kappa}^{ \pm} \boldsymbol{x}_{u_{i} u_{j}} .
$$

Since $-\mathbb{L}_{u_{i} u_{j}}^{ \pm}=-\mathbb{L}_{u_{j} u_{i}}^{ \pm}$and $\bar{\kappa}^{ \pm} \boldsymbol{x}_{u_{i} u_{j}}=\bar{\kappa}^{ \pm} \boldsymbol{x}_{u_{j} u_{i}}$, we have $\bar{\kappa}_{u_{j}}^{ \pm} \boldsymbol{x}_{u_{i}}-\bar{\kappa}_{u_{i}}^{ \pm} \boldsymbol{x}_{u_{j}}=0$. By definition, $\left\{\boldsymbol{x}_{u_{1}}, \ldots, \boldsymbol{x}_{u_{n-1}}\right\}$ is linearly independent, so that $\bar{\kappa}^{ \pm}$is constant. Since $\bar{\kappa}^{ \pm}=-1 \pm \kappa$, the condition $-\mathbb{L}_{u_{i}}^{ \pm}=\bar{\kappa}^{ \pm} \boldsymbol{x}_{u_{i}}$ is equivalent to the condition $-\boldsymbol{e}_{u_{i}}= \pm \kappa \boldsymbol{x}_{u_{i}}$.

We now assume that $\bar{\kappa}^{ \pm} \neq 0$. If $\bar{\kappa}^{ \pm} \neq-1$, then $\kappa \neq 0$, so that we have $\boldsymbol{x}_{u_{i}}= \pm(1 / \kappa) \boldsymbol{e}_{u_{i}}$. Therefore, there exists a constant vector $\boldsymbol{a}$ such that $\boldsymbol{x}=\boldsymbol{a} \mp(1 / \kappa) \boldsymbol{e}$. Since $\langle\boldsymbol{x}-\boldsymbol{a}, \boldsymbol{x}-\boldsymbol{a}\rangle=$ $(1 / \kappa)^{2}$, we have

$$
\langle\boldsymbol{a}, \boldsymbol{x}\rangle=-\frac{1}{2}\left(\frac{1}{\kappa^{2}}+1-\langle\boldsymbol{a}, \boldsymbol{a}\rangle\right)=-\frac{1}{2}\left(\frac{1}{\kappa^{2}}+1+1-\frac{1}{\kappa^{2}}\right)=-1 .
$$

This means that $M=\boldsymbol{x}(U) \subset H P(\boldsymbol{a},-1) \cap H_{+}^{n}(-1)$. If $|\kappa|<1, \boldsymbol{a}=\boldsymbol{x} \pm(1 / \kappa) \boldsymbol{e}$ is spacelike,so that we have the assertion 1)-a). In the case when $|\kappa|>1$ the assertion 1)-b) follows.

If $\bar{\kappa}^{ \pm}=-1$, then we have $e_{u_{i}}=0$. Thus $\boldsymbol{e}$ is a constant vector $\boldsymbol{a}$. Since $\boldsymbol{a}$ is a spacelike vector and we have $\langle\boldsymbol{x}, \boldsymbol{a}\rangle=0, M=\boldsymbol{x}(U) \subset H P(\boldsymbol{a}, 0) \cap H_{+}^{n}(-1)$. This means that $M$ is a part of a hyperplane.

Finally, we assume that $\bar{\kappa}^{ \pm}=0$. In this case, we have $-\mathbb{L}_{u_{i}}^{ \pm}=0$, so that $-\mathbb{L}^{ \pm}$is a constant lightlike vector $\boldsymbol{a}$. This means that the hyperbolic Gauss map is constant. The assertion 2) follows from Lemma 2.2. This completes the proof.

By the above proposition, we can classify the umbilic point as follows: Let $p=\boldsymbol{x}\left(u_{0}\right) \in$ $\boldsymbol{x}(U)=M$ be an umbilic point, we say that $p$ is an equidistant flat point if $\bar{\kappa}^{ \pm} \neq 0,0<$ $\left|\bar{\kappa}^{ \pm}+1\right|<1$, a hyperspherical point if $\bar{\kappa}^{ \pm} \neq 0,\left|\bar{\kappa}^{ \pm}+1\right|>1$, a flat point if $\bar{\kappa}^{ \pm} \neq 0,\left|\bar{\kappa}^{ \pm}+1\right|=0$, or a hyperhorospherical point if $\bar{\kappa}^{ \pm}=0$.

In the last part of this section, we prove the hyperbolic Weingarten formula: Since $\boldsymbol{x}_{u_{i}}$ $(i=1, \ldots n-1)$ are spacelike vectors, we induce the Riemannian metric (the hyperbolic first fundamental form ) $d s^{2}=\sum_{i=1}^{n-1} g_{i j} d u_{i} d u_{j}$ on $M=\boldsymbol{x}(U)$, where $g_{i j}(u)=\left\langle\boldsymbol{x}_{u_{i}}(u), \boldsymbol{x}_{u_{j}}(u)\right\rangle$ for any $u \in U$. We also define the hyperbolic second fundamental invariant by $\bar{h}_{i j}^{ \pm}(u)=$ $\left\langle-\mathbb{L}_{u_{i}}^{ \pm}(u), \boldsymbol{x}_{u_{j}}(u)\right\rangle$ for any $u \in U$. If we define the (ordinary) second fundamental invariant by $h_{i j}(u)=-\left\langle\boldsymbol{e}_{u_{i}}(u), \boldsymbol{x}_{u_{j}}(u)\right\rangle$, then we have the relation

$$
\bar{h}_{i j}^{ \pm}(u)=-g_{i j}(u) \pm h_{i j}(u) .
$$


Proposition 2.4 Under the above notations, we have the following hyperbolic Weingarten formula:

$$
\mathbb{L}_{u_{i}}^{ \pm}=-\sum_{j=1}^{n-1}\left(\bar{h}^{ \pm}\right)_{i}^{j} \boldsymbol{x}_{u_{j}}
$$

where $\left(\left(\bar{h}^{ \pm}\right)_{i}^{j}\right)=\left(\bar{h}_{i k}^{ \pm}\right)\left(g^{k j}\right)$ and $\left(g^{k j}\right)=\left(g_{k j}\right)^{-1}$.

Proof. There exist real numbers $\lambda, \mu, \Gamma_{i}^{j}$ such that

$$
\mathbb{L}_{u_{i}}^{ \pm}=\lambda e+\mu \boldsymbol{x}+\sum_{j=1}^{n-1} \Gamma_{i}^{j} \boldsymbol{x}_{u_{j}}
$$

Since $\left\langle\mathbb{L}^{ \pm}, \mathbb{L}^{ \pm}\right\rangle=0$, we have

$$
0=\left\langle\mathbb{L}_{u_{i}}^{ \pm}, \mathbb{L}^{ \pm}\right\rangle=\langle\lambda e+\mu \boldsymbol{x}, \boldsymbol{x} \pm e\rangle=-\mu \pm \lambda .
$$

Therefore, we have

$$
\mathbb{L}_{u_{i}}^{ \pm}=\mu \mathbb{L}^{ \pm}+\sum_{j=1}^{n-1} \Gamma_{i}^{j} \boldsymbol{x}_{u_{j}}
$$

By definition, we have

$$
-\bar{h}_{i \beta}^{ \pm}=\sum_{\alpha=1}^{n-1} \Gamma_{i}^{\alpha}\left\langle\boldsymbol{x}_{u_{\alpha}}, \boldsymbol{x}_{u_{\beta}}\right\rangle=\sum_{\alpha=1}^{n-1} \Gamma_{i}^{\alpha} g_{\alpha \beta}
$$

Hence, we have

$$
-\left(\bar{h}^{ \pm}\right)_{i}^{j}=-\sum_{\beta=1}^{n-1} \bar{h}_{i \beta}^{ \pm} g^{\beta j}=\sum_{\beta=1}^{n-1} \sum_{\alpha=1}^{n-1} \Gamma_{i}^{\alpha} g_{\alpha \beta} g^{\beta j}=\Gamma_{i}^{j} .
$$

On the other hand, since $\left\langle\mathbb{L}^{ \pm}, \boldsymbol{x}\right\rangle=-1$ and $\left\langle\mathbb{L}^{ \pm}, \boldsymbol{x}_{u_{i}}\right\rangle=0$, we have $\mu=-\mu\left\langle\mathbb{L}^{ \pm}, \boldsymbol{x}\right\rangle=$ $\left\langle\mathbb{L}_{u_{i}}^{ \pm}, \boldsymbol{x}\right\rangle=0$. This completes the proof of the hyperbolic Weingarten formula.

As a corollary of the above proposition, we have an explicit expression of the hyperbolic Gauss-Kronecker curvature by Riemannian metric and the hyperbolic second fundamental invariant.

Corollary 2.5 Under the same notations as in the above proposition, the hyperbolic GaussKronecker curvature is given by

$$
K_{h}^{ \pm}=\frac{\operatorname{det}\left(\bar{h}_{i j}^{ \pm}\right)}{\operatorname{det}\left(g_{\alpha \beta}\right)}
$$

Proof. By the hyperbolic Weingarten formula, the representation matrix of the hyperbolic shape operator with respect to the basis $\left\{\boldsymbol{x}_{u_{1}}, \ldots, \boldsymbol{x}_{u_{n-1}}\right\}$ is $\left(\left(\bar{h}^{ \pm}\right)_{i}^{j}\right)=\left(\bar{h}_{i \beta}^{ \pm}\right)\left(g^{\beta j}\right)$. It follows from this fact that

$$
K_{h}^{ \pm}=\operatorname{det} S_{p}^{ \pm}=\operatorname{det}\left(\left(\bar{h}^{ \pm}\right)_{i}^{j}\right)=\operatorname{det}\left(\bar{h}_{i \beta}^{ \pm}\right)\left(g^{\beta j}\right)=\frac{\operatorname{det}\left(\bar{h}_{i j}^{ \pm}\right)}{\operatorname{det}\left(g_{\alpha \beta}\right)}
$$

We say that a point $p=\boldsymbol{x}\left(u_{0}\right)$ is a (positive or negative) horospherical parabolic point (or, briefly a $H^{ \pm}$-parabolic point) of $\boldsymbol{x}: U \longrightarrow H_{+}^{n}(-1)$ if $K_{h}^{ \pm}\left(u_{0}\right)=0$. 


\section{Horospherical height functions}

In this section we introduce two different families of functions on a hypersurface in Hyperbolic space which are useful for the study of singularities of hyperbolic Gauss indicatrices and hyperbolic Gauss maps. Let $\boldsymbol{x}: U \longrightarrow H_{+}^{n}(-1)$ be a hypersurface. We define a family of functions

$$
H: U \times L C_{+}^{*} \longrightarrow \mathbb{R}
$$

by $H(u, \boldsymbol{v})=\langle\boldsymbol{x}(u), \boldsymbol{v}\rangle+1$. We call $H$ a horospherical ( or, a normalised lightcone) height function on $\boldsymbol{x}: U \longrightarrow H_{+}^{n}(-1)$.

Proposition 3.1 Let $H: U \times L C_{+}^{*} \longrightarrow \mathbb{R}$ be a horospherical height function on $\boldsymbol{x}: U \longrightarrow$ $H_{+}^{n}(-1)$. Then

(1) $H(u, v)=0$ if and only if there exist real numbers $\mu, \xi_{1}, \ldots, \xi_{n-1}$ such that $\boldsymbol{v}=\boldsymbol{x}+\mu \boldsymbol{e}+$ $\xi_{1} \boldsymbol{x}_{u_{1}}+\cdots+\xi_{n-1} \boldsymbol{x}_{u_{n-1}}$.

(2) $H(u, v)=\frac{\partial H}{\partial u_{i}}(u, v)=0(i=1, \ldots, n-1)$ if and only if $\boldsymbol{v}=\boldsymbol{x}(u) \pm \boldsymbol{e}(u)=\mathbb{L}^{ \pm}(u)$.

Proof. (1) Since $\left\{\boldsymbol{x}, \boldsymbol{e}, \boldsymbol{x}_{u_{1}}, \ldots, \boldsymbol{x}_{u_{n-1}}\right\}$ is a basis of the vector space $T_{p} \mathbb{R}_{1}^{n+1}$ where $\boldsymbol{p}=\boldsymbol{x}(u)$, there exist real numbers $\lambda, \mu, \xi_{1}, \ldots, \xi_{n-1}$ such that $\boldsymbol{v}=\lambda \boldsymbol{x}+\mu \boldsymbol{e}+\xi_{1} \boldsymbol{x}_{u_{1}}+\cdots+\xi_{n-1} \boldsymbol{x}_{u_{n-1}}$. Therefore $H(u, v)=0$ if and only if $-1=\langle\boldsymbol{x}, \boldsymbol{v}\rangle=\lambda\langle\boldsymbol{x}, \boldsymbol{x}\rangle=-\lambda$.

(2) Since $\frac{\partial H}{\partial u_{i}}(u, v)=\left\langle\boldsymbol{x}_{u_{i}}, \boldsymbol{v}\right\rangle$, we have $0=\left\langle\boldsymbol{x}_{u_{i}}, \boldsymbol{v}\right\rangle=\xi_{i}\left\langle\boldsymbol{x}_{u_{i}}, \boldsymbol{x}_{u_{i}}\right\rangle$. This means that the condition of (2) is equivalent to the condition $\boldsymbol{v}=\boldsymbol{x}+\mu \boldsymbol{e}$. Since $\langle\boldsymbol{v}, \boldsymbol{v}\rangle=0, \mu= \pm 1$. This completes the proof.

We denote the Hessian matrix of the horospherical height function $h_{v_{0}^{ \pm}}(u)=H\left(u, v_{0}^{ \pm}\right)$at $u_{0}$ by $\operatorname{Hess}\left(h_{v_{0}^{ \pm}}\right)\left(u_{0}\right)$.

Proposition 3.2 Let $\boldsymbol{x}: U \longrightarrow H_{+}^{n}(-1)$ be a hypersurface in Hyperbolic space and $\boldsymbol{v}_{0}^{ \pm}=$ $\mathbb{L}^{ \pm}\left(u_{0}\right)$. Then

(1) $p=\boldsymbol{x}\left(u_{0}\right)$ is a $H^{ \pm}$-parabolic point if and only if $\operatorname{det} \operatorname{Hess}\left(h_{v_{0}^{ \pm}}\right)\left(u_{0}\right)=0$.

(2) $p=\boldsymbol{x}\left(u_{0}\right)$ is a hyperhorospherical point if and only if $\operatorname{rank} \operatorname{Hess}\left(h_{v_{0}^{ \pm}}\right)\left(u_{0}\right)=0$.

Proof. By definition, we have

$$
\operatorname{Hess}\left(h_{v_{0}^{ \pm}}\right)\left(u_{0}\right)=\left(\left\langle\boldsymbol{x}_{u_{i} u_{j}}\left(u_{0}\right), \mathbb{L}^{ \pm}\left(u_{0}\right)\right\rangle\right)=\left(-\left\langle\boldsymbol{x}_{u_{i}}\left(u_{0}\right), \mathbb{L}_{u_{j}}^{ \pm}\left(u_{0}\right)\right\rangle\right)
$$

By the hyperbolic Weingarten formula, we have

$$
-\left\langle\boldsymbol{x}_{u_{i}}, \mathbb{L}_{u_{j}}^{ \pm}\right\rangle=\sum_{\alpha=1}^{n-1}\left(\bar{h}^{ \pm}\right)_{i}^{\alpha}\left\langle\boldsymbol{x}_{u_{\alpha}}, \boldsymbol{x}_{u_{j}}\right\rangle=\sum_{\alpha=1}^{n-1}\left(\bar{h}^{ \pm}\right)_{i}^{\alpha} g_{\alpha j}=\bar{h}_{i j}^{ \pm}
$$

Therefore we have

$$
K_{h}^{ \pm}\left(u_{0}\right)=\frac{\operatorname{det} \operatorname{Hess}\left(h_{v_{0}^{ \pm}}\right)\left(u_{0}\right)}{\operatorname{det}\left(g_{\alpha \beta}\left(u_{0}\right)\right)} .
$$

The first assertion follows from this formula. For the second assertion, by the hyperbolic Weingarten formula, $p=\boldsymbol{x}\left(u_{0}\right)$ is an umbilic point if and only if there exists an orthogonal matrix $A$ such that ${ }^{t} A\left(\left(\bar{h}^{ \pm}\right)_{i}^{\alpha}\right) A=\bar{\kappa}^{ \pm} I$. Therefore, we have $\left(\left(\bar{h}^{ \pm}\right)_{i}^{\alpha}\right)=A \bar{\kappa}^{ \pm} I^{t} A=\bar{\kappa}^{ \pm} I$, so that

$$
\operatorname{Hess}\left(h_{v_{0}^{ \pm}}\right)=\left(\bar{h}_{i j}^{ \pm}\right)=\left(\left(\bar{h}^{ \pm}\right)_{i}^{\alpha}\right)\left(g_{\alpha j}\right)=\bar{\kappa}^{ \pm}\left(g_{i j}\right) \text {. }
$$


Thus, $p$ is a hyperhorospherical point if and only if $\operatorname{rank} \operatorname{Hess}\left(h_{v_{0}^{ \pm}}\right)\left(u_{0}\right)=0$.

We also define a family of functions

$$
\bar{H}: U \times S_{+}^{n-1} \longrightarrow \mathbb{R}
$$

by $\bar{H}(u, \boldsymbol{v})=\langle\boldsymbol{x}(u), \boldsymbol{v}\rangle$. We call $\bar{H}$ a hyperbolic (or, a lightcone) height function on $\boldsymbol{x}: U \longrightarrow$ $H_{+}^{n}(-1)$.

Proposition 3.3 Let $\bar{H}: U \times S_{+}^{n-1} \longrightarrow \mathbb{R}$ be a hyperbolic height function on $\boldsymbol{x}: U \longrightarrow H_{+}^{n}(-1)$. Then $\frac{\partial \bar{H}}{\partial u_{i}}(u, \boldsymbol{v})=0(i=1, \ldots n-1)$ if and only if $\boldsymbol{v}=\widetilde{\mathbb{L}^{ \pm}}(u)$.

The proof is also given by the direct calculation like as those in Proposition 3.1.

\section{Hyperbolic Gauss indicatrices as wave fronts}

In this section we naturally interpret the hyperbolic Gauss indicatrix of a hypersurface in Hyperbolic space as a wave front set in the framework of contact geometry. We consider a point $\boldsymbol{v}=\left(v_{0}, v_{1}, \ldots, v_{n}\right) \in L C_{+}^{*}$, then we have the relation $v_{0}=\sqrt{v_{1}^{2}+\cdots+v_{n}^{2}}$. So we adopt the coordinate $\left(v_{1}, \ldots, v_{n}\right)$ of the manifold $L C_{+}^{*}$. Here, we consider the projective cotangent bundle $\pi: P T^{*}\left(L C_{+}^{*}\right) \longrightarrow L C_{+}^{*}$ with the canonical contact structure. We now review geometric properties of this space. Consider the tangent bundle $\tau: T P T^{*}\left(L C_{+}^{*}\right) \rightarrow P T^{*}\left(L C_{+}^{*}\right)$ and the differential map $d \pi: T P T^{*}\left(L C_{+}^{*}\right) \rightarrow T L C_{+}^{*}$ of $\pi$. For any $X \in T P T^{*}\left(L C_{+}^{*}\right)$, there exists an element $\alpha \in T^{*}\left(L C_{+}^{*}\right)$ such that $\tau(X)=[\alpha]$. For an element $V \in T_{x}\left(L C_{+}^{*}\right)$, the property $\alpha(V)=\mathbf{0}$ does not depend on the choice of representative of the class $[\alpha]$. Thus we can define the canonical contact structure on $P T^{*}\left(L C_{+}^{*}\right)$ by

$$
K=\left\{X \in T P T^{*}\left(L C_{+}^{*}\right) \mid \tau(X)(d \pi(X))=0\right\}
$$

Since we consider the coordinate $\left(v_{1}, \ldots, v_{n}\right)$, we have the trivialisation $P T^{*}\left(L C_{+}^{*}\right) \cong L C_{+}^{*} \times$ $P\left(\mathbb{R}^{n-1}\right)^{*}$, we call

$$
\left(\left(v_{1}, \ldots, v_{n}\right),\left[\xi_{1}: \cdots: \xi_{n}\right]\right)
$$

a homogeneous coordinate, where $\left[\xi_{1}: \cdots: \xi_{n}\right]$ is the homogeneous coordinate of the dual projective space $P\left(\mathbb{R}^{n-1}\right)^{*}$.

It is easy to show that $X \in K_{(x,[\xi])}$ if and only if $\sum_{i=1}^{n} \mu_{i} \xi_{i}=0$, where $d \tilde{\pi}(X)=\sum_{i=1}^{n} \mu_{i} \frac{\partial}{\partial v_{i}}$. An immersion $i: L \rightarrow P T^{*}\left(L C_{+}^{*}\right)$ is said to be a Legendrian immersion if $\operatorname{dim} L=n$ and $d i_{q}\left(T_{q} L\right) \subset K_{i(q)}$ for any $q \in L$. We also call the map $\pi \circ i$ the Legendrian map and the set $W(i)=$ image $\pi \circ i$ the wave front of $i$. Moreover, $i$ (or, the image of $i$ ) is called the Legendrian lift of $W(i)$.

For any hypersurface $\boldsymbol{x}: U \longrightarrow H_{+}^{n}(-1)$, we denote that $\boldsymbol{x}(u)=\left(x_{0}(u), \ldots, x_{n}(u)\right)$ and $\mathbb{L}^{ \pm}(u)=\left(\ell_{0}^{ \pm}(u), \ldots, \ell_{n}^{ \pm}(u)\right)$ as coordinate representations. We now define a smooth mapping

$$
\mathcal{L}^{ \pm}: U \longrightarrow P T^{*}\left(L C_{+}^{*}\right)
$$

by

$$
\mathcal{L}^{ \pm}(u)=\left(\mathbb{L}^{ \pm}(u),\left[-\ell_{1}^{ \pm}(u) x_{0}(u)+\ell_{0}^{ \pm}(u) x_{1}(u): \cdots:-\ell_{n}^{ \pm}(u) x_{0}+\ell_{0}^{ \pm}(u) x_{n}(u)\right]\right)
$$


Proposition $4.1 \mathcal{L}^{ \pm}$is an integral map with respect to the canonical contact structure. In other wards, we have $d \mathcal{L}_{u_{0}}^{ \pm}\left(T_{u_{0}} U\right) \subset K_{p}$ for any $u_{0} \in U$, where $p=\mathcal{L}^{ \pm}\left(u_{0}\right)$

Proof. For any $\frac{\partial}{\partial u_{i}} \in T_{u_{0}} U$, we have

$$
d \mathcal{L}^{ \pm}\left(\frac{\partial}{\partial u_{i}}\right)=\sum_{j=1}^{n} \frac{\partial \ell_{j}}{\partial u_{i}}\left(u_{0}\right) \frac{\partial}{\partial v_{j}}+V
$$

where $V$ is a tangent vector of the fibre of $\pi$. By definition, we have $\left\langle\boldsymbol{x}_{u_{i}}, \mathbb{L}^{ \pm}\right\rangle=0$. Since $\left\langle\boldsymbol{x}, \mathbb{L}^{ \pm}\right\rangle=-1$, we have $\left\langle\boldsymbol{x}, \mathbb{L}_{u_{i}}^{ \pm}\right\rangle=-\left\langle\boldsymbol{x}_{u_{i}}, \mathbb{L}^{ \pm}\right\rangle=0$. This means that

$$
-\ell_{0}^{ \pm} \frac{\partial x_{0}}{\partial u_{i}}+\ell_{1}^{ \pm} \frac{\partial x_{1}}{\partial u_{i}}+\cdots+\ell_{n}^{ \pm} \frac{\partial x_{n}}{\partial u_{i}}=0
$$

It also follows from the fact $\left\langle\mathbb{L}^{ \pm}, \mathbb{L}^{ \pm}\right\rangle=0$ that we have $\left\langle\mathbb{L}_{u_{i}}^{ \pm}, \mathbb{L}^{ \pm}\right\rangle=0$, so that

$$
\frac{\partial \ell_{0}^{ \pm}}{\partial u_{i}} \ell_{0}^{ \pm}=\sum_{j=1}^{n} \frac{\partial \ell_{j}^{ \pm}}{\partial u_{i}} \ell_{j}^{ \pm}
$$

Therefore, we have

$$
\sum_{j=1}^{n} \frac{\partial \ell_{j}^{ \pm}}{\partial u_{i}}\left(u_{0}\right)\left(-\ell_{j}^{ \pm}\left(u_{0}\right) x_{0}\left(u_{0}\right)+\ell_{0}^{ \pm}\left(u_{0}\right) x_{j}\left(u_{0}\right)\right)=\ell_{0}^{ \pm}\left(u_{0}\right)\left(\sum_{j=0}^{n}-x_{j}\left(u_{0}\right) \frac{\partial \ell_{j}^{ \pm}}{\partial u_{i}}\left(u_{0}\right)\right)=0 .
$$

We can show that $\mathcal{L}^{ \pm}$is always an immersion. We will show this fact in the next section. So we have the Legendrian lift $\mathcal{L}^{ \pm}$of $\mathbb{L}^{ \pm}$. The conclusion of this section is that the hyperbolic Gauss indicatrix of a hypersurface can be considered as a wave front.

\section{$5 \quad$ Generating families}

By the previous arguments, we can study singularities of Gauss indicatrices by the Legendrian singularity theory. For our purpose, we give a quick survey on the Legendrian singularity theory mainly due to Arnol'd-Zakalyukin[1, 25]. Almost all results have been known at least implicitly. Nevertheless, the topological theory for Legendrian singularities has not been written in any contexts except [11], so that we summarise here. Let $F:\left(\mathbb{R}^{k} \times \mathbb{R}^{n}, \mathbf{0}\right) \longrightarrow(\mathbb{R}, \mathbf{0})$ be a function germ. We say that $F$ is a Morse family if the mapping

$$
\Delta^{*} F=\left(F, \frac{\partial F}{\partial q_{1}}, \ldots, \frac{\partial F}{\partial q_{k}}\right):\left(\mathbb{R}^{k} \times \mathbb{R}^{n}, \mathbf{0}\right) \longrightarrow\left(\mathbb{R} \times \mathbb{R}^{k}, \mathbf{0}\right)
$$

is non-singular, where $(q, x)=\left(q_{1}, \ldots, q_{k}, x_{1}, \ldots, x_{n}\right) \in\left(\mathbb{R}^{k} \times \mathbb{R}^{n}, 0\right)$. In this case we have a smooth $(n-1)$-dimensional submanifold

$$
\Sigma_{*}(F)=\left\{(q, x) \in\left(\mathbb{R}^{k} \times \mathbb{R}^{n}, \mathbf{0}\right) \mid F(q, x)=\frac{\partial F}{\partial q_{1}}(q, x)=\cdots=\frac{\partial F}{\partial q_{k}}(q, x)=0\right\}
$$


and the map germ $\Phi_{F}:\left(\Sigma_{*}(F), \mathbf{0}\right) \longrightarrow P T^{*} \mathbb{R}^{n}$ defined by

$$
\Phi_{F}(q, x)=\left(x,\left[\frac{\partial F}{\partial x_{1}}(q, x): \cdots: \frac{\partial F}{\partial x_{n}}(q, x)\right]\right)
$$

is a Legendrian immersion. Then we have the following fundamental theorem of Arnol'dZakalyukin $[1,25]$.

Proposition 5.1 All Legendrian submanifold germs in $P T^{*} \mathbb{R}^{n}$ are constructed by the above method.

We call $F$ a generating family of $\Phi_{F}$. Therefore the wave front is

$W\left(\Phi_{F}\right)=\left\{x \in \mathbb{R}^{n} \mid\right.$ there exists $q \in \mathbb{R}^{k}$ such that $\left.F(q, x)=\frac{\partial F}{\partial q_{1}}(q, x)=\cdots=\frac{\partial F}{\partial q_{k}}(q, x)=0\right\}$.

We sometime denote that $\mathcal{D}_{F}=W\left(\Phi_{F}\right)$ and call it the discriminant set of $F$.

On the other hand, for any map $f: N \longrightarrow P$, we denote $\Sigma(f)$ the set of singular points of $f$ and $D(f)=f(\Sigma(f))$. In this case we call $f \mid \Sigma(f): \Sigma(f) \longrightarrow D(f)$ the critical part of the mapping $f$. For any Morse family $F:\left(\mathbb{R}^{k} \times \mathbb{R}^{n}, \mathbf{0}\right) \longrightarrow(\mathbb{R}, \mathbf{0}),\left(F^{-1}(0), \mathbf{0}\right)$ is a smooth hypersurface, so that we define a smooth map germ $\pi_{F}:\left(F^{-1}(0), \mathbf{0}\right) \longrightarrow(\mathbb{R}, 0)$ by $\pi_{F}(q, x)=x$. We can easily show that $\Sigma_{*}(F)=\Sigma\left(\pi_{F}\right)$. Therefore, the corresponding Legendrian map $\pi \circ \Phi_{F}$ is the critical part of $\pi_{F}$.

We now introduce an equivalence relation among Legendrian immersion germs. Let $i$ : $(L, p) \subset\left(P T^{*} \mathbb{R}^{n}, p\right)$ and $i^{\prime}:\left(L^{\prime}, p^{\prime}\right) \subset\left(P T^{*} \mathbb{R}^{n}, p^{\prime}\right)$ be Legendrian immersion germs. Then we say that $i$ and $i^{\prime}$ are Legendrian equivalent if there exists a contact diffeomorphism germ $H:\left(P T^{*} \mathbb{R}^{n}, p\right) \longrightarrow\left(P T^{*} \mathbb{R}^{n}, p^{\prime}\right)$ such that $H$ preserves fibres of $\pi$ and that $H(L)=L^{\prime}$. A Legendrian immersion germ into $P T^{*} \mathbb{R}^{n}$ at a point is said to be Legendrian stable if for every map with the given germ there is a neighbourhood in the space of Legendrian immersions (in the Whitney $C^{\infty}$ topology) and a neighbourhood of the original point such that each Legendrian immersion belonging to the first neighbourhood has in the second neighbourhood a point at which its germ is Legendrian equivalent to the original germ.

Since the Legendrian lift $i:(L, p) \subset\left(P T^{*} \mathbb{R}^{n}, p\right)$ is uniquely determined on the regular part of the wave front $W(i)$, we have the following simple but significant property of Legendrian immersion germs:

Proposition 5.2 Let $i:(L, p) \subset\left(P T^{*} \mathbb{R}^{n}, p\right)$ and $i^{\prime}:\left(L^{\prime}, p^{\prime}\right) \subset\left(P T^{*} \mathbb{R}^{n}, p^{\prime}\right)$ be Legendrian immersion germs such that regular sets of $\pi \circ i, \pi \circ i^{\prime}$ are dense respectively. Then $i, i^{\prime}$ are Legendrian equivalent if and only if wave front sets $W(i), W\left(i^{\prime}\right)$ are diffeomorphic as set germs.

This result has been firstly pointed out by Zakalyukin[26]. The assumption in the above proposition is a generic condition for $i, i^{\prime}$. Especially, if $i, i^{\prime}$ are Legendrian stable, then these satisfy the assumption.

We can interpret the Legendrian equivalence by using the notion of generating families. We denote $\mathcal{E}_{n}$ the local ring of function germs $\left(\mathbb{R}^{n}, \mathbf{0}\right) \longrightarrow \mathbb{R}$ with the unique maximal ideal $\mathfrak{M}_{n}=\left\{h \in \mathcal{E}_{n} \mid h(0)=0\right\}$. Let $F, G:\left(\mathbb{R}^{k} \times \mathbb{R}^{n}, \mathbf{0}\right) \longrightarrow(\mathbb{R}, \mathbf{0})$ be function germs. We say that $F$ and $G$ are $P$ - $\mathcal{K}$-equivalent if there exists a diffeomorphism germ $\Psi:\left(\mathbb{R}^{k} \times \mathbb{R}^{n}, \mathbf{0}\right) \longrightarrow\left(\mathbb{R}^{k} \times \mathbb{R}^{n}, \mathbf{0}\right)$ of the form $\Psi(x, u)=\left(\psi_{1}(q, x), \psi_{2}(x)\right)$ for $(q, x) \in\left(\mathbb{R}^{k} \times \mathbb{R}^{n}, \mathbf{0}\right)$ such that $\Psi^{*}\left(\langle F\rangle_{\mathcal{E}_{k+n}}\right)=\langle G\rangle_{\mathcal{E}_{k+n}}$. Here $\Psi^{*}: \mathcal{E}_{k+n} \longrightarrow \mathcal{E}_{k+n}$ is the pull back $\mathbb{R}$-algebra isomorphism defined by $\Psi^{*}(h)=h \circ \Psi$. 
Let $F:\left(\mathbb{R}^{k} \times \mathbb{R}^{3}, \mathbf{0}\right) \longrightarrow(\mathbb{R}, \mathbf{0})$ a function germ. We say that $F$ is a $\mathcal{K}$-versal deformation of $f=F \mid \mathbb{R}^{k} \times\{0\}$ if

$$
\mathcal{E}_{k}=T_{e}(\mathcal{K})(f)+\left\langle\frac{\partial F}{\partial x_{1}}\left|\mathbb{R}^{k} \times\{\mathbf{0}\}, \ldots, \frac{\partial F}{\partial x_{n}}\right| \mathbb{R}^{k} \times\{\mathbf{0}\}\right\rangle_{\mathbb{R}}
$$

where

(See [13].)

$$
T_{e}(\mathcal{K})(f)=\left\langle\frac{\partial f}{\partial q_{1}}, \ldots, \frac{\partial f}{\partial q_{k}}, f\right\rangle_{\mathcal{E}_{k}}
$$

The main result in Arnol'd-Zakalyukin's theory[1, 25] is as follows:

Theorem 5.3 Let $F, G:\left(\mathbb{R}^{k} \times \mathbb{R}^{n}, 0\right) \longrightarrow(\mathbb{R}, 0)$ be Morse families. Then

(1) $\Phi_{F}$ and $\Phi_{G}$ are Legendrian equivalent if and only if $F, G$ are $P$-K-equivalent.

(2) $\Phi_{F}$ is Legendrian stable if and only if $F$ is a $\mathcal{K}$-versal deformation of $F \mid \mathbb{R}^{k} \times\{\mathbf{0}\}$.

Since $F, G$ are function germs on the common space germ $\left(\mathbb{R}^{k} \times \mathbb{R}^{n}, \mathbf{0}\right)$, we do no need the notion of stably $P$ - $\mathcal{K}$-equivalences under this situation (cf., [1]). By the uniqueness result of the $\mathcal{K}$-versal deformation of a function germ, Proposition 5.2 and Theorem 5.3, we have the following classification result of Legendrian stable germs(cf., [10]). For any map germ $f:\left(\mathbb{R}^{n}, \mathbf{0}\right) \longrightarrow\left(\mathbb{R}^{p}, \mathbf{0}\right)$, we define the local ring of $f$ by $Q(f)=\mathcal{E}_{n} / f^{*}\left(\mathfrak{M}_{p}\right) \mathcal{E}_{n}$.

Proposition 5.4 Let $F, G:\left(\mathbb{R}^{k} \times \mathbb{R}^{n}, 0\right) \longrightarrow(\mathbb{R}, 0)$ be Morse families. Suppose that $\Phi_{F}, \Phi_{G}$ are Legendrian stable. The the following conditions are equivalent.

(1) $\left(W\left(\Phi_{F}\right), \mathbf{0}\right)$ and $\left(W\left(\Phi_{G}\right), \mathbf{0}\right)$ are diffeomorphic as germs.

(2) $\Phi_{F}$ and $\Phi_{G}$ are Legendrian equivalent.

(3) $Q(f)$ and $Q(g)$ are isomorphic as $\mathbb{R}$-algebras, where $f=F\left|\mathbb{R}^{k} \times\{\mathbf{0}\}, g=G\right| \mathbb{R}^{k} \times\{\mathbf{0}\}$.

Proof. Since $\Phi_{F}, \Phi_{G}$ are Legendrian stable, these satisfy the generic condition of Proposition 5.2 , so that the conditions (1) and (2) are equivalent. The condition (3) implies that $f, g$ are $\mathcal{K}$-equivalent[13, 14]. By the uniqueness of the $\mathcal{K}$-versal deformation of a function germ, $F, G$ are $P-\mathcal{K}$-equivalent. This means that the condition (2) holds. By Theorem 5.3, the condition (2) implies the condition (3).

We now consider what does the wave front look like generically? We have another characterisation of $\mathcal{K}$-versal deformations of function germs. Let $J^{\ell}\left(\mathbb{R}^{k}, \mathbb{R}\right)$ be the $\ell$-jet bundle of $n$-variable functions which has the canonical decomposition: $J^{\ell}\left(\mathbb{R}^{k}, \mathbb{R}\right) \equiv \mathbb{R}^{k} \times \mathbb{R} \times J^{\ell}(k, 1)$. For any Morse family $F:\left(\mathbb{R}^{k} \times \mathbb{R}^{n}, \mathbf{0}\right) \longrightarrow(\mathbb{R}, 0)$, we define a map germ

$$
j_{1}^{\ell} F:\left(\mathbb{R}^{k} \times \mathbb{R}^{n}, \mathbf{0}\right) \longrightarrow J^{\ell}\left(\mathbb{R}^{k}, \mathbb{R}\right)
$$

by $j_{1}^{\ell} F(q, x)=j^{\ell} F_{x}(q)$, where $F_{x}(q)=F(q, x)$. We denote $\mathcal{K}^{\ell}(z)$ the $\mathcal{K}$-orbit through $z=$ $j^{\ell} f(\mathbf{0}) \in J^{\ell}(k, 1)$. (cf., [13]). If $f(q)=F(q, \mathbf{0})$ is $\ell$-determined relative to $\mathcal{K}$, then $F$ is a $\mathcal{K}$-versal deformation of $f$ if and only if $j_{1}^{\ell} F$ is transversal to $\mathbb{R}^{k} \times\{0\} \times \mathcal{K}^{\ell}(z)$ (cf., [13])

We now consider the stratification such that the discriminant set of $\mathcal{K}$-versal deformations has the corresponding canonical stratification. By Theorem 5.3, such a stratification should be $\mathcal{K}$-invariant, where we have the $\mathcal{K}$-action on $J^{\ell}(k, 1)$ (cf., $[13,14]$ ). By this reason, we use 
Mather's canonical stratification here $[9,15]$. Let $\mathcal{A}^{\ell}(k, 1)$ be the canonical stratification of $J^{\ell}(k, 1) \backslash W^{\ell}(k, 1)$, where

$$
W^{\ell}(k, 1)=\left\{j^{\ell} f(0) \mid \operatorname{dim}_{\mathbb{R}} \mathcal{E}_{k} /\left(\left(T_{e} \mathcal{K}\right)(f)+\mathfrak{M}_{k}^{\ell}\right) \geq \ell\right\} .
$$

We now define the stratification $\mathcal{A}_{0}^{\ell}\left(\mathbb{R}^{k}, \mathbb{R}\right)$ of $J^{\ell}\left(\mathbb{R}^{k}, \mathbb{R}\right) \backslash W^{\ell}\left(\mathbb{R}^{k}, \mathbb{R}\right)$ by

$$
\mathbb{R}^{k} \times(\mathbb{R} \backslash\{0\}) \times\left(J^{\ell}(k, 1) \backslash W^{\ell}(k, 1)\right), \mathbb{R}^{k} \times\{0\} \times \mathcal{A}^{\ell}(k, 1),
$$

where $W^{\ell}\left(\mathbb{R}^{k}, \mathbb{R}\right) \equiv \mathbb{R}^{k} \times \mathbb{R} \times W^{\ell}(k, 1)$. In [22], Y.-H. Wan has shown that if $j_{1}^{\ell} F(0) \notin W^{\ell}(k, 1)$ and $j_{1}^{\ell} F$ is transversal to $\mathcal{A}_{0}^{\ell}\left(\mathbb{R}^{k}, \mathbb{R}\right)$ then $\pi_{F}:\left(F^{-1}(0), \mathbf{0}\right) \longrightarrow\left(\mathbb{R}^{n}, \mathbf{0}\right)$ is a MT-stable map germ. (See also [11]). Here, we call a map germ $M T$-stable if it is transversal to the canonical stratification of a jet space which is introduced in $[9,15]$. The main assertion of Mather's topological stability theorem is that an MT-stable map germ is a topological stable map germ. Moreover, the critical value of an MT-stable map germ is canonically stratified. For the classification, we refer the following theorem of Fukuda-Fukuda[8].

Theorem 5.5 Let $f, g:\left(\mathbb{R}^{n}, 0\right) \longrightarrow\left(\mathbb{R}^{p}, \mathbf{0}\right)$ be $M T$-stable map germs. If $Q(f)$ and $Q(g)$ are isomorphic as $\mathbb{R}$-algebras, then these map germs are topological equivalent.

If we carefully read their proof, we can conclude that critical value sets (discriminant sets) of $f, g$ are stratified equivalent. Here we say that two stratified sets are stratified equivalent if there exists a homeomorphism between stratified sets such that the homeomorphism maps a strata onto a strata and the restriction on each strata is smooth.

In order to apply Theorem 5.5 to our situation, we need to review the theory of unfoldings of map germs. The definition of $r$-dimensional unfolding of $f:\left(\mathbb{R}^{n}, 0\right) \longrightarrow\left(\mathbb{R}^{p}, 0\right)$ (originally due to Thom) is a germ $\widetilde{F}:\left(\mathbb{R}^{n} \times \mathbb{R}^{r}, 0\right) \longrightarrow\left(\mathbb{R}^{p} \times \mathbb{R}^{r}, 0\right)$ given by $\widetilde{F}(x, u)=(F(x, u), u)$, where $F(x, u)$ is a germ of $r$ dimensional parametrised families of germs with $F(x, 0)=f(x)$. This definition depends on the coordinates of both of spaces $\left(\mathbb{R}^{n} \times \mathbb{R}^{r}, 0\right)$ and $\left(\mathbb{R}^{p} \times \mathbb{R}^{r}, 0\right)$. For our purpose, we need the coordinate free definition of unfoldings[9]. Let $f:\left(N, x_{0}\right) \longrightarrow\left(P, y_{0}\right)$ be a map germ between manifolds. An unfolding of $f$ is a triple $(\widetilde{F}, i, j)$ of map germs, where $i:\left(N, x_{0}\right) \longrightarrow\left(N^{\prime}, x_{0}^{\prime}\right), j:\left(P, y_{0}\right) \longrightarrow\left(P^{\prime}, y_{0}^{\prime}\right)$ are immersions and $j$ is transverse to $\widetilde{F}$, such that $\widetilde{F} \circ i=j \circ f$ and $(i, f): N \longrightarrow\left\{\left(x^{\prime}, y\right) \in N^{\prime} \times P \mid \widetilde{F}\left(x^{\prime}\right)=j(y)\right\}$ is a diffeomorphism germ. The dimension of $(\widetilde{F}, i, j)$ as an unfolding is $\operatorname{dim} N^{\prime}-\operatorname{dim} N$. We can easily prove that the above two definitions are equivalent. We can show that the local ring of a map germ does not depend on the choice of the local coordinates at the points. Therefore we can define the local ring $Q\left(\pi_{F}\right)$ for a Morse family $F$. We can easily show that $Q(f)$ and $Q(\widetilde{F})$ are canonically isomorphic as $\mathbb{R}$-algebras.

We now apply the above arguments to our case. The idea used here is originally from Martinet's study of stable map germs[13]. Let $F:\left(\mathbb{R}^{k} \times \mathbb{R}^{n}, 0\right) \longrightarrow(\mathbb{R}, 0)$ be a Morse family. Corresponding to $F$, we have an unfolding of $f=F \mid\{0\} \times \mathbb{R}^{n}$

$$
\widetilde{F}:\left(\mathbb{R}^{k} \times \mathbb{R}^{n}, \mathbf{0}\right) \longrightarrow\left(\mathbb{R} \times \mathbb{R}^{n}, \mathbf{0}\right)
$$

given by $\widetilde{F}(q, x)=(F(q, x), x)$. Then we can easily show the following lemma.

Lemma 5.6 We consider inclusions $i:\left(F^{-1}(0), \mathbf{0}\right) \longrightarrow\left(\mathbb{R}^{k} \times \mathbb{R}^{n}, \mathbf{0}\right)$ and $j:\left(\{0\} \times \mathbb{R}^{n}, \mathbf{0}\right) \longrightarrow$ $\left(\mathbb{R} \times \mathbb{R}^{n}, \mathbf{0}\right)$. Then $(\widetilde{F}, i, j)$ is an unfolding of $\pi_{F}:\left(F^{-1}(0), \mathbf{0}\right) \longrightarrow\left(\mathbb{R}^{n}, \mathbf{0}\right)$. 
By the previous arguments, $Q\left(\pi_{F}\right), Q(\widetilde{F})$ and $Q(f)$ are isomorphic to each other as $\mathbb{R}$ algebras. By Theorem 5.5, we have the following proposition:

Proposition 5.7 Let $F, G:\left(\mathbb{R}^{k} \times \mathbb{R}^{n}, 0\right) \longrightarrow(\mathbb{R}, 0)$ be Morse families such that $\pi_{F}$ and $\pi_{G}$ are $M T$-stable map germs. If $Q(f)$ and $Q(g)$ are isomorphic as $\mathbb{R}$-algebras, then $\pi_{F}$ and $\pi_{G}$ are topological equivalent. Moreover, in this case, $\mathcal{D}_{F}$ and $\mathcal{D}_{G}$ are stratified equivalent.

Let $F:\left(\mathbb{R}^{n} \times \mathbb{R}^{n}, \mathbf{0}\right) \longrightarrow(\mathbb{R}, 0)$ be a Morse family. Suppose that $j_{1}^{\ell} F(\mathbf{0}) \notin W^{\ell}(k, 1)$ and $j_{1}^{\ell} F$ is transversal to $\mathcal{A}_{0}^{\ell}\left(\mathbb{R}^{k}, \mathbb{R}\right)$ for sufficient large $\ell$ (i.e., codim $\left.W^{\ell}(k, 1)>k+n\right)$. By the transversality assumption, we cannot avoid strata $X_{j}$ of codimension $\leq k+n$. For $n \leq 6$ and $\ell \geq 8$, by the classification of $\mathcal{K}$-simple function germs [1], $\operatorname{codim} W^{\ell}(k, 1)>k+6$ and each strata of $\mathcal{A}^{\ell}(k, 1)$ is a $\mathcal{K}^{\ell}$-orbit in $J^{\ell}(k, 1)$. In this case, we can say that $F$ is a $\mathcal{K}$-versal deformation of $f=F \mid \mathbb{R}^{k} \times\{\mathbf{0}\}$ by the characterisation of $\mathcal{K}$-versal deformations. Therefore $\Phi_{F}$ is Legendrian stable. For general $n \geq 7$, by the previous arguments, the wave front $W\left(\Phi_{F}\right)$ is the discriminant set of the MT-stable map germ $\pi_{F}:\left(F^{-1}(0), \mathbf{0}\right) \longrightarrow\left(\mathbb{R}^{n}, \mathbf{0}\right)$.

In the last part of this section we return to the study of the horospherical height function of a hypersurface in $H_{+}^{n}(-1)$. By Proposition 4.1, we have the Legendrian lift $\mathcal{L}^{ \pm}$of the hyperbolic Gauss indicatrix $\mathbb{L}^{ \pm}$. Since we have the following proposition, Proposition 3.1 means that the horospherical height function $H: U \times L C_{0}^{*} \longrightarrow \mathbb{R}$ is the generating family of $\mathcal{L}^{ \pm}$.

Proposition 5.8 The horospherical height function $H: U \times L C_{0}^{*} \longrightarrow \mathbb{R}$ is a Morse family.

Proof. For any $\boldsymbol{v}=\left(v_{0}, v_{1}, \ldots, v_{n}\right) \in L C_{+}^{*}$, we have $v_{0}=\sqrt{v_{1}^{2}+\cdots+v_{n}^{2}}$, so that

$$
H(u, \boldsymbol{v})=-x_{0}(u) \sqrt{v_{1}^{2}+\cdots+v_{n}^{2}}+x_{1}(u) v_{1}+\cdots+x_{n}(u) v_{n}+1
$$

where $\boldsymbol{x}(u)=\left(x_{0}(u), \ldots, x_{n}(u)\right)$. We have to prove that the mapping

$$
\Delta^{*} H=\left(H, \frac{\partial H}{\partial u_{1}}, \ldots, \frac{\partial H}{\partial u_{n-1}}\right)
$$

is non-singular at any point. The Jacobian matrix of $\Delta^{*} H$ is given as follows:

$$
\left(\begin{array}{cccccc}
\left\langle\boldsymbol{x}_{u_{1}}, \boldsymbol{v}\right\rangle & \cdots & \left\langle\boldsymbol{x}_{u_{n-1}}, \boldsymbol{v}\right\rangle & -x_{0} \frac{v_{1}}{v_{0}}+x_{1} & \cdots & -x_{0} \frac{v_{n}}{v_{0}}+x_{n} \\
\left\langle\boldsymbol{x}_{u_{1} u_{1}}, \boldsymbol{v}\right\rangle & \cdots & \left\langle\boldsymbol{x}_{u_{1} u_{n-1}}, \boldsymbol{v}\right\rangle & -x_{0 u_{1}} \frac{v_{1}}{v_{0}}+x_{1 u_{1}} & \cdots & -x_{0 u_{1}} \frac{v_{n}}{v_{0}}+x_{n u_{1}} \\
\vdots & \vdots & \vdots & \vdots & \vdots & \vdots \\
\left\langle\boldsymbol{x}_{u_{n-1} u_{1}}, \boldsymbol{v}\right\rangle & \cdots & \left\langle\boldsymbol{x}_{u_{n-1} u_{n-1}}, \boldsymbol{v}\right\rangle & -x_{0 u_{n-1}} \frac{v_{1}}{v_{0}}+x_{1 u_{n-1}} & \cdots & -x_{0 u_{n-1}} \frac{v_{n}}{v_{0}}+x_{n u_{n-1}}
\end{array}\right)
$$

We now show that the determinant of the matrix

$$
A=\left(\begin{array}{ccc}
-x_{0} \frac{v_{1}}{v_{0}}+x_{1} & \cdots & -x_{0} \frac{v_{n}}{v_{0}}+x_{n} \\
-x_{0 u_{1}} \frac{v_{1}}{v_{0}}+x_{1 u_{1}} & \cdots & -x_{0 u_{1}} \frac{v_{n}}{v_{0}}+x_{n u_{1}} \\
\vdots & \vdots & \vdots \\
-x_{0 u_{n-1}} \frac{v_{1}}{v_{0}}+x_{1 u_{n-1}} & \cdots & -x_{0 u_{n-1}} \frac{v_{n}}{v_{0}}+x_{n u_{n-1}}
\end{array}\right) .
$$


does not vanish at $(u, v) \in \Sigma_{*}(H)$. In this case, $\boldsymbol{v}=\mathbb{L}^{ \pm}(u)$ and we denote that

$$
\boldsymbol{a}=\left(\begin{array}{c}
x_{0} \\
x_{0 u_{1}} \\
\vdots \\
x_{0 u_{n-1}}
\end{array}\right), \boldsymbol{b}_{1}=\left(\begin{array}{c}
x_{1} \\
x_{1 u_{1}} \\
\vdots \\
x_{1 u_{n-1}}
\end{array}\right), \ldots, \boldsymbol{b}_{n}=\left(\begin{array}{c}
x_{n} \\
x_{n u_{1}} \\
\vdots \\
x_{n u_{n-1}}
\end{array}\right)
$$

Then we have

$$
\operatorname{det} A=\frac{v_{0}}{v_{0}} \operatorname{det}\left(\boldsymbol{b}_{1} \ldots \boldsymbol{b}_{n}\right)-\frac{v_{1}}{v_{0}} \operatorname{det}\left(\boldsymbol{a} \boldsymbol{b}_{2} \ldots \boldsymbol{b}_{n}\right)-\cdots-\frac{v_{n}}{v_{0}} \operatorname{det}\left(\boldsymbol{b}_{1} \ldots \boldsymbol{b}_{n-1} \boldsymbol{a}\right) .
$$

On the other hand, we have

$\boldsymbol{x} \wedge \boldsymbol{x}_{u_{1}} \wedge \cdots \wedge \boldsymbol{x}_{u_{n-1}}=\left(-\operatorname{det}\left(\boldsymbol{b}_{1} \ldots \boldsymbol{b}_{n}\right),-\operatorname{det}\left(\boldsymbol{a}_{\boldsymbol{b}_{2}} \ldots \boldsymbol{b}_{n}\right), \ldots,-\operatorname{det}\left(\boldsymbol{b}_{1} \ldots \boldsymbol{b}_{n-1} \boldsymbol{a}\right)\right)$.

Therefore we have

$$
\operatorname{det} A=\left\langle\left(\frac{v_{0}}{v_{0}}, \ldots, \frac{v_{n}}{v_{0}}\right), \boldsymbol{x} \wedge \boldsymbol{x}_{u_{1}} \wedge \cdots \wedge \boldsymbol{x}_{u_{n-1}}\right\rangle=\frac{1}{v_{0}}\left\langle\mathbb{L}^{ \pm}, \boldsymbol{e}\right\rangle= \pm \frac{1}{v_{0}} \neq 0
$$

We have the following corollary which we announced in $\S 4$.

Corollary 5.9 For any hypersurface $\boldsymbol{x}: U \longrightarrow H_{+}^{n}(-1), \mathcal{L}^{ \pm}$is a Legendrian immersion whose generating family is the horospherical height function $H: U \times L C_{+}^{*} \longrightarrow \mathbb{R}$ of $\boldsymbol{x}$.

\section{Duality and hyperbolic Gauss maps}

In $\S 2$ we call $\mathbb{L}^{ \pm}$the hyperbolic Gauss indicatrix or the lightcone dual of $\boldsymbol{x}$. In the first part of this section we describe why we call it the lightcone dual of $\boldsymbol{x}$. After that we consider the relationship between the hyperbolic Gauss indicatrix and the hyperbolic Gauss map of a hypersurface. We consider a function $H^{*}: H_{+}^{n}(-1) \times U \longrightarrow \mathbb{R}$ defined by $H^{*}(\boldsymbol{v}, u)=\left\langle\boldsymbol{v}, \mathbb{L}^{ \pm}(u)\right\rangle+1$. Since $\mathbb{L}^{ \pm}(U)$ is a wave front set, we have the tangent space $V_{p}$ at any point $p=\mathbb{L}^{ \pm}(u)$. By Lemma 2.1, $V_{p}$ is equal to the tangent space $T_{q} \boldsymbol{x}(U)$ for $q=\boldsymbol{x}(u)$, so that $V_{p}$ is an $(n-1)$-dimensional spacelike subspace of $\mathbb{R}_{1}^{n+1}$. Therefore, we have the intersection curve $V_{p}^{\perp} \cap H_{+}^{n}(-1)$. Since $\mathbb{L}^{ \pm}(u) \in L C_{+}^{*}$, there exists a unique $\boldsymbol{v} \in V_{p}^{\perp} \cap H_{+}^{n}(-1)$ such that $\left\langle\mathbb{L}^{ \pm}(u), \boldsymbol{v}\right\rangle=-1$. We denote that $\left(\mathbb{L}^{ \pm}\right)^{*}(u)=v$, then we have a mapping

$$
\left(\mathbb{L}^{ \pm}\right)^{*}: U \longrightarrow H_{+}^{n}(-1) \text {. }
$$

If $u \in U$ is a regular point of $\mathbb{L}^{ \pm}$, then $\left(\mathbb{L}^{ \pm}\right)^{*}(u)$ satisfies the conditions that

$$
\left\langle\left(\mathbb{L}^{ \pm}\right)^{*}(u), \mathbb{L}^{ \pm}(u)\right\rangle=-1,\left\langle\left(\mathbb{L}^{ \pm}\right)^{*}(u),\left(\mathbb{L}^{ \pm}\right)_{u_{i}}(u)\right\rangle=0 .
$$

This means that $\left(\mathbb{L}^{ \pm}\right)^{*}(u) \in \mathcal{D}_{H^{*}}$.

On the other hand, since $\boldsymbol{x}$ and $\mathbb{L}^{ \pm}$satisfy the condition that

$$
\left\langle\boldsymbol{x}, \mathbb{L}^{ \pm}\right\rangle=-1,\left\langle\boldsymbol{x}_{u_{i}}, \mathbb{L}^{ \pm}\right\rangle=0
$$

we have $\left\langle\boldsymbol{x}, \mathbb{L}_{u_{i}}^{ \pm}\right\rangle=0$. Hence, we have $\boldsymbol{x}=\left(\mathbb{L}^{ \pm}\right)^{*}$ at a regular point of $\mathbb{L}^{ \pm}$. Since both of mappings are defined on $U$ and $\left(\mathbb{L}^{ \pm}\right)^{*}$ is at least a continuous mapping, we have the following proposition. 
Proposition 6.1 Suppose that the $H^{ \pm}$-parabolic set of $\boldsymbol{x}: U \longrightarrow H_{+}^{n}(-1)$ has no inner point, then we have $\left(\mathbb{L}^{ \pm}\right)^{*}=\boldsymbol{x}$.

We remark that the assumption of the above proposition is generic for $x: U \longrightarrow H_{+}^{n}(-1)$.

On the other hand, if we start a spacelike immersion $y: U \longrightarrow L C_{+}^{*}$ and consider a function $H^{\sharp}: H_{+}^{n}(-1) \times U \longrightarrow \mathbb{R}$ defined by $H^{\sharp}(\boldsymbol{v}, u)=\langle\boldsymbol{v}, \boldsymbol{y}(u)\rangle+1$, we can obtain the dual mapping $\boldsymbol{y}^{*}: U \longrightarrow H_{+}^{n}(-1)$ of $\boldsymbol{y}$ exactly the same way as the previous arguments. If $\boldsymbol{y}^{*}$ is a immersion at $u \in U$, we can easily show that one of the hyperbolic Gauss indicatrix of $\boldsymbol{y}^{*}$ at $u$ is $\boldsymbol{y}(u)$. have

If we consider a function $\mathcal{H}: H_{+}^{n}(-1) \times L C_{+}^{*} \longrightarrow \mathbb{R}$ defined by $\mathcal{H}\left(\boldsymbol{v}_{1}, \boldsymbol{v}_{2}\right)=\left\langle\boldsymbol{v}_{1}, \boldsymbol{v}_{2}\right\rangle+1$, we

$$
H=\mathcal{H} \circ\left(\boldsymbol{x} \times i d_{L C_{+}^{*}}\right), H^{*}=\mathcal{H} \circ\left(i d_{H_{+}^{n}(-1)} \times \mathbb{L}^{ \pm}\right) \text {and } H^{\sharp}=\mathcal{H} \circ\left(i d_{H_{+}^{n}(-1)} \times \boldsymbol{y}\right) .
$$

We understand that all arguments in this section are controlled by the function $\mathcal{H}$. As a conclusion, we may call $\mathbb{L}^{ \pm}$the lightcone dual of $\boldsymbol{x}: U \longrightarrow H_{+}^{n}(-1)$.

On the other hand, we now consider the relation between the hyperbolic Gauss indicatrix and the hyperbolic Gauss map of a hypersurface in $H_{+}^{n}(-1)$. For any $\boldsymbol{x}: U \longrightarrow H_{+}^{n}(-1)$, we define a function $\mathfrak{H}: U \times S_{+}^{n-1} \longrightarrow \mathbb{R}$ by $\mathfrak{H}(u, \boldsymbol{v})=\langle\boldsymbol{x}(u), \boldsymbol{v}\rangle$. We call $\mathfrak{H}$ the lightcone height function of $\boldsymbol{x}$. We also define a function $\widetilde{\mathfrak{H}}: U \times S_{+}^{n-1} \times \mathbb{R}_{+} \longrightarrow \mathbb{R}$ by $\widetilde{\mathfrak{H}}(u, \boldsymbol{v}, y)=\mathfrak{H}(u, \boldsymbol{v})+y=\langle\boldsymbol{x}(u), \boldsymbol{v}\rangle+y$, where $\mathbb{R}_{+}$denotes the set of positive real numbers. We call $\widetilde{\mathfrak{H}}$ the extended lightcone height function on $\boldsymbol{x}$. By the similar calculations like as the proof of Proposition 3.1, we have

$$
\mathcal{D}_{\widetilde{\mathfrak{H}}}=\left\{\left(\widetilde{\mathbb{L}^{ \pm}}(u),-\left\langle\boldsymbol{x}(u), \widetilde{\mathbb{L}^{ \pm}}(u)\right\rangle\right) \in S_{+}^{n-1} \times \mathbb{R}_{+} \mid u \in U\right\} .
$$

Let $\pi_{1}: S_{+}^{n-1} \times \mathbb{R} \longrightarrow S_{+}^{n-1}$ be the canonical projection, then $\pi_{1} \mid \mathcal{D}_{\tilde{\mathfrak{H}}}$ can be identified to the hyperbolic Gauss map of $\boldsymbol{x}$.

We define a diffeomorphism $\Phi: S_{+}^{n-1} \times \mathbb{R}_{+} \longrightarrow L C_{+}^{*}$ by $\Phi(v, y)=\frac{1}{y} v$. Since

$$
\mathbb{L}^{ \pm}(u)=-\frac{1}{\left\langle\boldsymbol{x}(u), \mathbb{L}^{ \pm}(u)\right\rangle} \widetilde{\mathbb{L}^{ \pm}}(u),
$$

we have $\Phi\left(\mathcal{D}_{\tilde{\mathfrak{H}}}\right)=\left\{\mathbb{L}^{ \pm}(u) \mid u \in U\right\}=\mathcal{D}_{H}$. By these arguments, we can say that the hyperbolic Gauss indicatrix is a lift of the hyperbolic Gauss map. In fact, by the general theory of Lagrangian and Legendrian mappings $[1,25]$, we can construct the Lagrangian immersion $\widetilde{\mathcal{L}^{ \pm}}: U \longrightarrow T^{*} S_{+}^{n-1}$ such that $\Pi \circ \widetilde{\mathcal{L}^{ \pm}}=\widetilde{\mathbb{L}^{ \pm}}$whose generating family is the lightcone height function $\mathfrak{H}$, where $\Pi: T^{*} S_{+}^{n-1} \longrightarrow S_{+}^{n-1}$ is the projection of the cotangent bundle over $S_{+}^{n-1}$. This means that the hyperbolic Gauss map is a Lagrangian map like as ordinary Gauss maps in Euclidean space.

On the other hand, we also have a Legendrian immersion $\mathfrak{L}^{ \pm}: U \longrightarrow T^{*} S_{+}^{n-1} \times \mathbb{R}_{+}$given by $\mathfrak{L}^{ \pm}(u)=\left(\widetilde{\mathcal{L}^{ \pm}}(u),-\left\langle\boldsymbol{x}(u), \mathbb{L}^{ \pm}(u)\right\rangle\right)$ which covers $\widetilde{\mathcal{L}^{ \pm}}$. The generating family of $\mathfrak{L}^{ \pm}$is the extended lightcone height function $\mathfrak{H}$. We can naturally regard that $T^{*} S_{+}^{n-1} \times \mathbb{R}_{+}$is an affine part of $P T^{*}\left(S_{+}^{n-1} \times \mathbb{R}_{+}\right)$. We also have a relation $H \circ\left(i d_{U} \times \Phi\right)(u, \boldsymbol{v}, y)=-\frac{1}{y} \widetilde{\mathfrak{H}}(u, \boldsymbol{v}, y)$ which means that $H \circ\left(i d_{U} \times \Phi\right)$ and $\tilde{\mathfrak{H}}$ are $\mathcal{C}$-equivalent in the sense of Mather[14]. It has been known that these generating families correspond to a same Legendrian submanifold $[1,25]$. In this case we have a unique contact diffeomorphism $\hat{\Phi}: P T^{*}\left(S_{+}^{n-1} \times \mathbb{R}_{+}\right) \longrightarrow P T^{*}\left(L C_{+}^{*}\right)$ covering $\Phi: S_{+}^{n-1} \times \mathbb{R}_{+} \longrightarrow L C_{+}^{*}$ such that $\hat{\Phi} \circ \mathfrak{L}^{ \pm}=\mathcal{L}^{ \pm}$, so that $\mathcal{L}^{ \pm}$is considered to be a covering of $\widetilde{\mathcal{L}^{ \pm}}$. 
This is the background relation between the hyperbolic Gauss indicatrix and the hyperbolic Gauss map of a hypersurface in $H_{+}^{n}(-1)$. Moreover, we have the relation

$$
\Phi^{-1} \circ \mathbb{L}^{ \pm}(u)=\left(\widetilde{\mathbb{L}^{ \pm}}(u),-\left\langle\boldsymbol{x}(u), \mathbb{L}^{ \pm}(u)\right\rangle\right),
$$

so that

$$
d\left(\Phi^{-1} \circ \mathbb{L}^{ \pm}(u)\right)=d\left(\widetilde{\mathbb{L}^{ \pm}}(u)\right) \oplus d\left(-\left\langle\boldsymbol{x}(u), \mathbb{L}^{ \pm}(u)\right\rangle\right)
$$

By Lemma 2.2, we have

$$
\left(-\left\langle\boldsymbol{x}(u), \mathbb{L}^{ \pm}(u)\right\rangle\right)_{u_{i}}=-\left\langle\boldsymbol{x}_{u_{i}}(u), \mathbb{L}^{ \pm}(u)\right\rangle-\left\langle\boldsymbol{x}(u), d \mathbb{L}^{ \pm}(u)_{u_{i}}\right\rangle=0 .
$$

This means that $d\left(-\left\langle\boldsymbol{x}(u), \mathbb{L}^{ \pm}(u)\right\rangle=\mathbf{0}\right.$. Thus we have the following result.

Proposition 6.2 The set of singular points of the hyperbolic Gauss map of a hypersurface is coincide with the $H^{ \pm}$-parabolic set of the hypersurface. In other wards, we have

$$
\Sigma\left(\widetilde{\mathbb{L}^{ \pm}}\right)=\Sigma\left(\mathbb{L}^{ \pm}\right)=\left\{u \in U \mid K_{h}^{ \pm}(u)=0\right\}
$$

\section{Contact with hyperhorospheres}

Before we start to consider the contact between hypersurfaces and hyperhorospheres, we briefly review the theory of contact due to Montaldi[18]. Let $X_{i}, Y_{i}(i=1,2)$ be submanifolds of $\mathbb{R}^{n}$ with $\operatorname{dim} X_{1}=\operatorname{dim} X_{2}$ and $\operatorname{dim} Y_{1}=\operatorname{dim} Y_{2}$. We say that the contact of $X_{1}$ and $Y_{1}$ at $y_{1}$ is same type as the contact of $X_{2}$ and $Y_{2}$ at $y_{2}$ if there is a diffeomorphism germ $\Phi:\left(\mathbb{R}^{n}, y_{1}\right) \longrightarrow\left(\mathbb{R}^{n}, y_{2}\right)$ such that $\Phi\left(X_{1}\right)=X_{2}$ and $\Phi\left(Y_{1}\right)=Y_{2}$. In this case we write $K\left(X_{1}, Y_{1} ; y_{1}\right)=K\left(X_{2}, Y_{2} ; y_{2}\right)$. It is clear that in the definition $\mathbb{R}^{n}$ could be replaced by any manifold. In his paper[18], Montaldi gives a characterisation of the notion of contact by using the terminology of singularity theory.

Theorem 7.1 Let $X_{i}, Y_{i}(i=1,2)$ be submanifolds of $\mathbb{R}^{n}$ with $\operatorname{dim} X_{1}=\operatorname{dim} X_{2}$ and $\operatorname{dim} Y_{1}=$ $\operatorname{dim} Y_{2}$. Let $g_{i}:\left(X_{i}, x_{i}\right) \longrightarrow\left(\mathbb{R}^{n}, y_{i}\right)$ be immersion germs and $f_{i}:\left(\mathbb{R}^{n}, y_{i}\right) \longrightarrow\left(\mathbb{R}^{p}, 0\right)$ be submersion germs with $\left(Y_{i}, y_{i}\right)=\left(f_{i}^{-1}(0), y_{i}\right)$. Then $K\left(X_{1}, Y_{1} ; y_{1}\right)=K\left(X_{2}, Y_{2} ; y_{2}\right)$ if and only if $f_{1} \circ g_{1}$ and $f_{2} \circ g_{2}$ are $\mathcal{K}$-equivalent.

We now remember the function $\mathcal{H}: H_{+}^{n}(-1) \times L C_{+}^{*} \longrightarrow \mathbb{R}$ given in $\S 6$. For any $\boldsymbol{v}_{0} \in L C_{+}^{*}$, we denote that $\mathfrak{h}_{v_{0}}(\boldsymbol{u})=\mathcal{H}\left(\boldsymbol{u}, \boldsymbol{v}_{0}\right)$ and we have a hyperhorosphere $\mathfrak{h}_{v_{0}}^{-1}(0)=H P\left(\boldsymbol{v}_{0},-1\right) \cap H_{+}^{n}(-1)$. We write that $H S\left(\boldsymbol{v}_{0},-1\right)=H P\left(\boldsymbol{v}_{0},-1\right) \cap H_{+}^{n}(-1)$. For any $u_{0} \in U$, we consider the lightlike vector $\boldsymbol{v}_{0}^{ \pm}=\mathbb{L}^{ \pm}\left(u_{0}\right)$, then we have

$$
\mathfrak{h}_{v_{0}^{ \pm}} \circ \boldsymbol{x}\left(u_{0}\right)=\mathcal{H} \circ\left(\boldsymbol{x} \times i d_{L C_{+}^{*}}\right)\left(u_{0}, \boldsymbol{v}_{0}^{ \pm}\right)=H\left(u_{0}, \mathbb{L}^{ \pm}\left(u_{0}\right)\right)=0 .
$$

We also have relations that

$$
\frac{\partial \mathfrak{h}_{v_{0}^{ \pm}} \circ \boldsymbol{x}}{\partial u_{i}}\left(u_{0}\right)=\frac{\partial H}{\partial u_{i}}\left(u_{0}, \mathbb{L}^{ \pm}\left(u_{0}\right)\right)=0 .
$$

for $i=1, \ldots, n-1$. This means that the hyperhorosphere $\mathfrak{h}_{v_{0}^{ \pm}}^{-1}(0)=H S\left(\boldsymbol{v}_{0}^{ \pm},-1\right)$ is tangent to $M=\boldsymbol{x}(U)$ at $p=\boldsymbol{x}\left(u_{0}\right)$. In this case, we call $H S\left(\boldsymbol{v}_{0}^{ \pm},-1\right)$ the tangent hyperhorosphere of $M=$ $\boldsymbol{x}(U)$ at $p=\boldsymbol{x}\left(u_{0}\right)$ (or, $\left.u_{0}\right)$, which we write $H S^{ \pm}\left(\boldsymbol{x}, u_{0}\right)$. Let $\boldsymbol{v}_{1}, \boldsymbol{v}_{2}$ be lightlike vectors. If $\boldsymbol{v}_{1}, \boldsymbol{v}_{2}$ are linearly dependent, then corresponding lightlike hyperplanes $\operatorname{HP}\left(\boldsymbol{v}_{1},-1\right), H P\left(\boldsymbol{v}_{2},-1\right)$ are parallel. Therefore, we say that two hyperhorospheres $H S\left(\boldsymbol{v}_{1},-1\right), H S\left(\boldsymbol{v}_{2},-1\right)$ are parallel if $\boldsymbol{v}_{1}, \boldsymbol{v}_{2}$ are linearly dependent. Then we have the following simple lemma. 
Lemma 7.2 Let $\boldsymbol{x}: U \longrightarrow H_{+}^{n}(-1)$ be a hypersurface. Consider two points $u_{1}, u_{2} \in U$. Then

(1) $\mathbb{L}^{ \pm}\left(u_{1}\right)=\mathbb{L}^{ \pm}\left(u_{2}\right)$ if and only if $H S^{ \pm}\left(\boldsymbol{x}, u_{1}\right)=H S^{ \pm}\left(\boldsymbol{x}, u_{2}\right)$.

(2) $\widetilde{\mathbb{L}^{ \pm}}\left(u_{1}\right)=\widetilde{\mathbb{L}^{ \pm}}\left(u_{2}\right)$ if and only if $H S^{ \pm}\left(\boldsymbol{x}, u_{1}\right), H S^{ \pm}\left(\boldsymbol{x}, u_{2}\right)$ are parallel.

Eventually, we have tools for the study of the contact between hypersurfaces and hyperhorospheres.

Let $\mathbb{L}_{i}^{ \pm}:\left(U, u_{i}\right) \longrightarrow\left(L C_{+}^{*}, \boldsymbol{v}_{i}^{ \pm}\right)(i=1,2)$ be two hyperbolic Gaussian indicatrix germs of hypersurface germs $\boldsymbol{x}_{i}:\left(U, u_{i}\right) \longrightarrow\left(H_{+}^{n}(-1), \boldsymbol{x}_{i}\left(u_{i}\right)\right)$. We say that $\mathbb{L}_{1}^{ \pm}$and $\mathbb{L}_{2}^{ \pm}$are $\mathcal{A}$-equivalent if there exist diffeomorphism germs $\phi:\left(U, u_{1}\right) \longrightarrow\left(U, u_{2}\right)$ and $\Phi:\left(L C_{+}^{*}, \boldsymbol{v}_{1}^{ \pm}\right) \longrightarrow\left(L C_{+}^{*}, \boldsymbol{v}_{2}^{ \pm}\right)$ such that $\Phi \circ \mathbb{L}_{1}^{ \pm}=\mathbb{L}_{2}^{ \pm} \circ \phi$. If the both of the regular sets of $\mathbb{L}_{i}^{ \pm}$are dense in $\left(U, u_{i}\right)$, it follows from Proposition 5.2 that $\mathbb{L}_{1}^{ \pm}$and $\mathbb{L}_{2}^{ \pm}$are $\mathcal{A}$-equivalent if and only if the corresponding Legendrian immersion germs $\mathcal{L}_{1}^{ \pm}:\left(U, u_{1}\right) \longrightarrow\left(P T^{*}\left(L C_{+}^{*}\right), z_{1}^{ \pm}\right)$and $\mathcal{L}_{1}^{ \pm}:\left(U, u_{2}\right) \longrightarrow\left(P T^{*}\left(L C_{+}^{*}\right), z_{2}^{ \pm}\right)$are Legendrian equivalent. This condition is also equivalent to the condition that two generating families $H_{1}$ and $H_{2}$ are $P$ - $\mathcal{K}$-equivalent by Theorem 5.3. Here, $H_{i}:\left(U \times L C_{+}^{*},\left(u_{i}, \boldsymbol{v}_{i}^{ \pm}\right)\right) \longrightarrow \mathbb{R}$ is the horospherical height function germ of $\boldsymbol{x}_{i}$.

On the other hand, we denote that $h_{i, v_{i}^{ \pm}}(u)=H_{i}\left(u, v_{i}^{ \pm}\right)$, then we have $h_{i, v_{i}^{ \pm}}(u)=\mathfrak{h}_{v_{i}^{ \pm}} \circ \boldsymbol{x}_{i}(u)$. By Theorem 7.1, $K\left(\boldsymbol{x}_{1}(U), H S^{ \pm}\left(\boldsymbol{x}_{1}, u_{1}\right), \boldsymbol{v}_{1}^{ \pm}\right)=K\left(\boldsymbol{x}_{2}(U), H S^{ \pm}\left(\boldsymbol{x}_{2}, u_{2}\right), \boldsymbol{v}_{2}^{ \pm}\right)$if and only if $h_{1, v_{1}}$ and $h_{1, v_{2}}$ are $\mathcal{K}$-equivalent. Therefore, we can apply the arguments in $\S 5$ to our situation. We denote $Q^{ \pm}\left(\boldsymbol{x}, u_{0}\right)$ the local ring of the function germ $h_{v_{0}^{ \pm}}:\left(U, u_{0}\right) \longrightarrow \mathbb{R}$, where $\boldsymbol{v}_{0}^{ \pm}=\mathbb{L}^{ \pm}\left(u_{0}\right)$. We remark that we can explicitly write the local ring as follows:

$$
Q^{ \pm}\left(\boldsymbol{x}, u_{0}\right)=\frac{C_{u_{0}}^{\infty}(U)}{\left\langle\left\langle\boldsymbol{x}(u), \mathbb{L}^{ \pm}\left(u_{0}\right)\right\rangle+1\right\rangle_{C_{u_{0}}^{\infty}(U)}}
$$

where $C_{u_{0}}^{\infty}(U)$ is the local ring of function germs at $u_{0}$ with the unique maximal ideal $\mathfrak{M}_{u_{0}}(U)$.

Theorem 7.3 Let $\boldsymbol{x}_{i}:\left(U, u_{i}\right) \longrightarrow\left(H_{+}^{n}(-1), \boldsymbol{x}_{i}\left(u_{i}\right)\right)(i=1,2)$ be hypersurfaces germs such that the corresponding Legendrian immersion germs $\mathcal{L}_{i}^{ \pm}:\left(U, u_{i}\right) \longrightarrow\left(P T^{*}\left(L C_{+}^{*}\right), z_{i}^{ \pm}\right)$are Legendrian stable. Then the following conditions are equivalent:

(1) Hyperbolic Gauss indicatrix germs $\mathbb{L}_{1}^{ \pm}$and $\mathbb{L}_{2}^{ \pm}$are $\mathcal{A}$-equivalent.

(2) $\mathrm{H}_{1}$ and $\mathrm{H}_{2}$ are $P$-K-equivalent.

(3) $h_{1, v_{1}}$ and $h_{1, v_{2}}$ are $\mathcal{K}$-equivalent.

(4) $K\left(\boldsymbol{x}_{1}(U), H S^{ \pm}\left(\boldsymbol{x}_{1}, u_{1}\right), \boldsymbol{v}_{1}^{ \pm}\right)=K\left(\boldsymbol{x}_{2}(U), H S^{ \pm}\left(\boldsymbol{x}_{2}, u_{2}\right), \boldsymbol{v}_{2}^{ \pm}\right)$

(5) $Q^{ \pm}\left(\boldsymbol{x}_{1}, u_{1}\right)$ and $Q^{ \pm}\left(\boldsymbol{x}_{2}, u_{2}\right)$ are isomorphic as $\mathbb{R}$-algebras.

Proof. By the previous arguments (mainly from Theorem 7.1), it has been already shown that conditions (3) and (4) are equivalent. Other assertions follow from Proposition 5.4.

In the next section, we will prove that the assumption of the theorem is generic in the case when $n \leq 6$. For general dimension, we need the topological theory.

Theorem 7.4 Let $\boldsymbol{x}_{i}:\left(U, u_{i}\right) \longrightarrow\left(H_{+}^{n}(-1), \boldsymbol{x}_{i}\left(u_{i}\right)\right)(i=1,2)$ be hypersurfaces germs such that the map germ given by $\pi_{H_{i}}:\left(H_{i}^{-1}\left(\boldsymbol{v}_{i}^{ \pm}\right),\left(u_{i}, \boldsymbol{v}_{i}^{ \pm}\right)\right) \longrightarrow\left(L C_{+}^{*}, \boldsymbol{v}_{i}^{ \pm}\right)$at any point $u_{i} \in U$ is an MT-stable map germ, where $H_{i}$ is the horospherical height function of $\boldsymbol{x}_{i}$ and $\boldsymbol{v}_{i}^{ \pm}=\mathbb{L}_{i}^{ \pm}\left(u_{i}\right)$. If $Q^{ \pm}\left(\boldsymbol{x}_{1}, u_{1}\right)$ and $Q^{ \pm}\left(\boldsymbol{x}_{2}, u_{2}\right)$ are isomorphic as $\mathbb{R}$-algebras, then $\left(\mathbb{L}_{1}^{ \pm}(U), u_{1}\right)$ and $\left(\mathbb{L}_{2}^{ \pm}(U), u_{2}\right)$ are stratified equivalent as set germs.

In general we have the following proposition. 
Proposition 7.5 Let $\boldsymbol{x}_{i}:\left(U, u_{i}\right) \longrightarrow\left(H_{+}^{n}(-1), \boldsymbol{x}_{i}\left(u_{i}\right)\right)(i=1,2)$ be hypersurface germs such that their $H^{ \pm}$-parabolic sets have no innerpoints as subspaces of $U$. If hyperbolic Gauss indicatrix germs $\mathbb{L}_{1}^{ \pm}, \mathbb{L}_{2}^{ \pm}$are $\mathcal{A}$-equivalent, then

$$
K\left(\boldsymbol{x}_{1}(U), H S^{ \pm}\left(\boldsymbol{x}_{1}, u_{1}\right), \boldsymbol{v}_{1}^{ \pm}\right)=K\left(\boldsymbol{x}_{2}(U), H S^{ \pm}\left(\boldsymbol{x}_{2}, u_{2}\right), \boldsymbol{v}_{2}^{ \pm}\right) .
$$

In this case, $\left(\boldsymbol{x}_{1}^{-1}\left(H S\left(\mathbb{L}_{1}^{ \pm}\left(u_{1}\right),-1\right)\right), u_{1}\right)$ and $\left(\boldsymbol{x}_{2}^{-1}\left(H S\left(\mathbb{L}_{2}^{ \pm}\left(u_{2}\right),-1\right)\right), u_{2}\right)$ are diffeomorphic as set germs.

Proof. The $H^{ \pm}$-parabolic set is the set of singular points of the hyperbolic Gauss indicatrix. So the corresponding Legendrian lifts $\mathcal{L}_{i}^{ \pm}$satisfy the hypothesis of Proposition 5.2. If hyperbolic Gauss indicatrix germs $\mathbb{L}_{1}^{ \pm}, \mathbb{L}_{2}^{ \pm}$are $\mathcal{A}$-equivalent, then $\mathcal{L}_{1}^{ \pm}, \mathcal{L}_{2}^{ \pm}$are Legendrian equivalent, so that $H_{1}, H_{2}$ are $P$ - $\mathcal{K}$-equivalent. Therefore, $h_{1, v_{1}}, h_{1, v_{2}}$ are $\mathcal{K}$-equivalent. By Theorem 7.1, this condition is equivalent to the condition that $K\left(\boldsymbol{x}_{1}(U), H S^{ \pm}\left(\boldsymbol{x}_{1}, u_{1}\right), \boldsymbol{v}_{1}^{ \pm}\right)=$ $K\left(\boldsymbol{x}_{2}(U), H S^{ \pm}\left(\boldsymbol{x}_{2}, u_{2}\right), \boldsymbol{v}_{2}^{ \pm}\right)$.

On the other hand, we have $\left(\boldsymbol{x}_{i}^{-1}\left(H S\left(\mathbb{L}_{i}^{ \pm}\left(u_{0}\right),-1\right)\right), u_{0}\right)=\left(h_{i, v_{i}}^{-1}(0), u_{0}\right)$. It follows from this fact that $\left(\boldsymbol{x}_{1}^{-1}\left(H S\left(\mathbb{L}_{1}^{ \pm}\left(u_{1}\right),-1\right)\right), u_{1}\right)$ and $\left(\boldsymbol{x}_{2}^{-1}\left(H S\left(\mathbb{L}_{2}^{ \pm}\left(u_{2}\right),-1\right)\right), u_{2}\right)$ are diffeomorphic as set germs because the $\mathcal{K}$-equivalence preserve the zero level sets.

For a hypersurface germ $\boldsymbol{x}:\left(U, u_{0}\right) \longrightarrow\left(H_{+}^{n}(-1), \boldsymbol{x}\left(u_{0}\right)\right)$, we call $\left(\boldsymbol{x}^{-1}\left(H S\left(\mathbb{L}^{ \pm}\left(u_{0}\right),-1\right)\right), u_{0}\right)$ the tangent hyperhorospherical indicatrix germ of $\boldsymbol{x}$. By Proposition 7.5 , the diffeomorphism type of the tangent hyperhorospherical indicatrix germ is an invariant of the $\mathcal{A}$-classification of the hyperbolic Gauss indicatrix germ of $\boldsymbol{x}$. Moreover, by the above results, we can borrow some basic invariants from the singularity theory on function germs. We need $\mathcal{K}$-invariants for function germ. The local ring of a function germ is a complete $\mathcal{K}$-invariant for generic function germs. It is, however, not a numerical invariant. The $\mathcal{K}$-codimension (or, Tyurina number) of a function germ is a numerical $\mathcal{K}$-invariant of function germs[13]. We denote that

$$
\mathrm{H}-\operatorname{ord}^{ \pm}\left(\boldsymbol{x}, u_{0}\right)=\operatorname{dim} \frac{C_{u_{0}}^{\infty}(U)}{\left\langle\left\langle\boldsymbol{x}(u), \mathbb{L}^{ \pm}\left(u_{0}\right)\right\rangle+1,\left\langle\boldsymbol{x}_{u_{i}}(u), \mathbb{L}^{ \pm}\left(u_{0}\right)\right\rangle\right\rangle_{C_{u_{0}}}} .
$$

Usually H-ord ${ }^{ \pm}\left(\boldsymbol{x}, u_{0}\right)$ is called the $\mathcal{K}$-codimension of $h_{v_{0}^{ \pm}}$. However, we call it the order of contact with the tangent hyperhorosphere at $\boldsymbol{x}\left(u_{0}\right)$. We also have the notion of corank of function germs.

$$
\mathrm{H}-\operatorname{corank}^{ \pm}\left(\boldsymbol{x}, u_{0}\right)=(n-1)-\operatorname{rank} \operatorname{Hess}\left(h_{v_{0}^{ \pm}}\left(u_{0}\right)\right) \text {, }
$$

where $v_{0}=\mathbb{L}^{ \pm}\left(u_{0}\right)$.

By Proposition $3.2, \boldsymbol{x}\left(u_{0}\right)$ is a $H^{ \pm}$-parabolic point if and only if $\mathrm{H}$-corank ${ }^{ \pm}\left(\boldsymbol{x}, u_{0}\right) \geq 1$. Moreover $\boldsymbol{x}\left(u_{0}\right)$ is a hyperhorospherical point if and only if $\mathrm{H}$-corank ${ }^{ \pm}\left(\boldsymbol{x}, u_{0}\right)=n-1$.

On the other hand, a function germ $f:\left(\mathbb{R}^{n-1}, \boldsymbol{a}\right) \longrightarrow \mathbb{R}$ has the $A_{k}$-type singularity if $f$ is $\mathcal{K}$ equivalent to the germ $\pm u_{1}^{2} \pm \cdots \pm u_{n-2}^{2}+u_{n-1}^{k+1}$. If $H$-corank ${ }^{ \pm}\left(\boldsymbol{x}, u_{0}\right)=1$, the horospherical height function $h_{v_{0}^{ \pm}}$has the $A_{k^{-}}$type singularity at $u_{0}$ in generic. In this case we have $\mathrm{H}$-ord ${ }^{ \pm}\left(\boldsymbol{x}, u_{0}\right)=k$. This number is equal to the order of contact in the classical sense (cf., [5]). This is the reason why we call $\mathrm{H}$-ord ${ }^{ \pm}\left(\boldsymbol{x}, u_{0}\right)$ the order of contact with the tangent hyperhorosphere at $\boldsymbol{x}\left(u_{0}\right)$.

\section{Generic properties}

In this section we consider generic properties of hypersurfaces in $H_{+}^{n}(-1)$. The main tool is a kind of transversality theorems. We consider the space of proper embeddings $\operatorname{Emb}\left(U, H_{+}^{n}(-1)\right)$ 
with Whitney $C^{\infty}$-topology. We also consider the function $\mathcal{H}: H_{+}^{n}(-1) \times L C_{+}^{*} \longrightarrow \mathbb{R}$ which is given in $\S 6$. We claim that $\mathcal{H}_{u}$ is a submersion for any $\boldsymbol{u} \in L C_{+}^{*}$, where $\mathcal{H}_{u}(\boldsymbol{v})=\mathcal{H}(\boldsymbol{u}, \boldsymbol{v})$. For any $\boldsymbol{x} \in \operatorname{Emb}\left(U, H_{+}^{n}(-1)\right)$, we have $H=\mathcal{H} \circ\left(\boldsymbol{x} \times i d_{L C_{+}^{*}}\right)$. We also have the $\ell$-jet extension

$$
j_{1}^{\ell} H: U \times L C_{+}^{*} \longrightarrow J^{\ell}(U, \mathbb{R})
$$

defined by $j_{1}^{\ell} H(u, v)=j^{\ell} h_{v}(u)$. We consider the trivialisation $J^{\ell}(U, \mathbb{R}) \equiv U \times \mathbb{R} \times J^{\ell}(n-1,1)$. For any submanifold $Q \subset J^{\ell}(n-1,1)$, we denote that $\widetilde{Q}=U \times\{0\} \times Q$. Then we have the following proposition as a corollary of Lemma 6 in Wassermann[23]. (See also Montaldi[19]).

Proposition 8.1 Let $Q$ be a submanifold of $J^{\ell}(n-1,1)$. Then the set

$$
T_{Q}=\left\{\boldsymbol{x} \in \operatorname{Emb}\left(U, H_{+}^{n}(-1)\right) \mid j_{1}^{\ell} H \text { is transversal to } \widetilde{Q}\right\}
$$

is a residual subset of $\operatorname{Emb}\left(U, H_{+}^{n}(-1)\right)$. If $Q$ is a closed subset, then $T_{Q}$ is open:

On the other hand, we already have the canonical stratification $\mathcal{A}_{0}^{\ell}(U ; \mathbb{R})$ of $J^{\ell}\left(\mathbb{R}^{n-1}, \mathbb{R}\right) \backslash$ $W^{\ell}\left(\mathbb{R}^{n-1}, \mathbb{R}\right)$. By the above proposition and arguments in $\S 5$, we have the following theorem.

Theorem 8.2 There exists an open dense subset $\mathcal{O} \subset \operatorname{Emb}\left(U, H_{+}^{n}(-1)\right)$ such that for any $\boldsymbol{x} \in \mathcal{O}$, the germ of the corresponding hyperbolic Gauss indicatrix $\mathbb{L}^{ \pm}$at each point is the critical part of an $M T$-stable map germ.

In the case when $n \leq 6$, for any $\boldsymbol{x} \in \mathcal{O}$, the germ of the Legendrian lift $\mathcal{L}^{ \pm}$of the hyperbolic Gauss indicatrix at each point is Legendrian stable.

We remark that we can also prove the multi-jet version of Proposition 8.1. As an application of such a multi-jet transversality theorem, we can show that the hyperbolic Gauss indicatrix is the critical part of an (global) MT-stable map for a generic hypersurface $x: U \longrightarrow H_{+}^{n}(-1)$. However, the arguments are rather tedious and we only consider local phenomenon in this paper, so that we omit it.

\section{Hyperbolic Monge form}

The notion of the Monge form of a hypersurface in Euclidean space is one of the powerful tools for the study of local properties of the hypersurface from the view point of differential geometry. In this section we consider the analogous notion for a hypersurface in Hyperbolic space.

We now consider a function $f\left(u_{1}, \ldots, u_{n-1}\right)$ with $f(0)=0$ and $f_{u_{i}}(0)=0$. Then we have a hypersurface in $H_{+}^{n}(-1)$ defined by

$$
\boldsymbol{x}_{f}\left(u_{1}, \ldots, u_{n-1}\right)=\left(\sqrt{f^{2}\left(u_{1}, \ldots, u_{n-1}\right)+u_{1}^{2}+\cdots+u_{n-1}^{2}+1}, f\left(u_{1}, \ldots, u_{n-1}\right), u_{1}, \ldots, u_{n-1}\right) .
$$

We can easily calculate that $\boldsymbol{e}(0)=(0,1,0 \ldots, 0)$, therefore $\mathbb{L}^{ \pm}(0)=(1, \pm 1,0, \ldots, 0)$. We call $\boldsymbol{x}_{f}$ a hyperbolic Monge form (briefly, H-Monge form). Then we have the following proposition.

Proposition 9.1 Any hypersurface in $H_{+}^{n}(-1)$ is locally given by the H-Monge form.

Proof. Let $\boldsymbol{x}: U \longrightarrow H_{+}^{n}(-1)$ be a hypersurface. We can apply Lorentzian motions of Minkowski space $\mathbb{R}_{1}^{n+1}$ such that $H_{+}^{n}(-1)$ is the invariant set. Therefore, without loss of generality, we assume that $p=\boldsymbol{x}(0)=(1,0, \ldots, 0)$. We denote $M=\boldsymbol{x}(U)$. We have a basis 
$\left\{\boldsymbol{x}(0), \boldsymbol{e}(0), \boldsymbol{x}_{u_{1}}(0), \ldots, \boldsymbol{x}_{u_{n-1}}(0)\right\}$ of $T_{p} \mathbb{R}_{1}^{n+1}$ such that $T_{p} M=\left\langle\boldsymbol{x}_{u_{1}}(0), \ldots, \boldsymbol{x}_{u_{n-1}}(0)\right\rangle_{\mathbb{R}}$. Applying the Gramm-Schmidt procedure, we have a pseudo-orthonormal basis $\left\{\boldsymbol{x}(0), \boldsymbol{e}(0), \boldsymbol{e}_{1}, \ldots \boldsymbol{e}_{n-1}\right\}$ of $\mathbb{R}_{1}^{n+1}$ such that $T_{p} M=\left\langle\boldsymbol{e}_{1}, \ldots \boldsymbol{e}_{n-1}\right\rangle_{\mathbb{R}}$. Especially $\left\{\boldsymbol{e}_{1}, \ldots, \boldsymbol{e}_{n-1}\right\}$ is an orthonormal basis of $T_{p} M$. Since $p=(1,0, \ldots, 0), T_{p} M$ is considered to be a subspace of $\mathbb{R}_{0}^{n}=\left\{\left(0, x_{1}, \ldots, x_{n}\right) \mid x_{i} \in \mathbb{R}\right\}$. By a rotation of the space $\mathbb{R}_{0}^{n}$, we might assume that $T_{p} M=\left\{\left(0,0, u_{1}, \ldots, u_{n-1}\right) \mid u_{i} \in \mathbb{R}\right\} \subset \mathbb{R}_{1}^{n+1}$. Then the germ $(M, p)$ might be written in the form

$$
\left(f_{0}\left(u_{1}, \ldots, u_{n-1}\right), f\left(u_{1}, \ldots, u_{n-1}\right), u_{1}, \ldots, u_{n-1}\right)
$$

by function germs $f_{0}\left(u_{1}, \ldots, u_{n-1}\right), f\left(u_{1}, \ldots, u_{n-1}\right)$. Since $M \subset H_{+}^{n}(-1)$, we have the relation

$$
f_{0}\left(u_{1}, \ldots, u_{n-1}\right)=\sqrt{f^{2}\left(u_{1}, \ldots, u_{n-1}\right)+u_{1}^{2}+\cdots+u_{n-1}^{2}+1} .
$$

Since we have $T_{p} M=\left\{\left(0,0, u_{1}, \ldots, u_{n-1}\right) \mid u_{i} \in \mathbb{R}\right\}$, the conditions $f(0)=0$ and $f_{u_{i}}(0)=0$ are automatically satisfied. This completes the proof.

For the lightlike vector $\boldsymbol{v}_{0}^{ \pm}=(1, \pm 1,0, \ldots, 0)$, we consider the hyperhorosphere $H S\left(\boldsymbol{v}_{0}^{ \pm},-1\right)$. Then we have the H-Monge form of $H S\left(\boldsymbol{v}_{0}^{ \pm},-1\right)$ :

$$
h^{ \pm}\left(u_{1}, \ldots, u_{n-1}\right)=\left(\frac{u_{1}^{2}+\cdots+u_{n-1}^{2}+2}{2}, \pm \frac{u_{1}^{2}+\cdots+u_{n-1}^{2}}{2}, u_{1}, \ldots, u_{n-1}\right) .
$$

Here, we can easily check the relation that $\left\langle\boldsymbol{v}_{0}^{ \pm}, \boldsymbol{h}^{ \pm}(u)\right\rangle=-1$.

On the other hand, $\boldsymbol{h}^{ \pm}(0)=(1,0, \ldots, 0)=p$ and $\boldsymbol{h}_{u_{i}}^{ \pm}(0)$ is equal to the $x_{i+1}$-axis for $i=1,2, \ldots, n-1$. This means that $T_{p} M=T_{p}\left(\boldsymbol{h}^{ \pm}(U)\right)$. Therefore $\boldsymbol{h}^{ \pm}(U)=H S\left(\boldsymbol{v}_{0},-1\right)$ is the tangent hyperhorosphere of $M=x_{f}(U)$ at $p=x_{f}(0)$. It follows from this fact that the tangent hyperhorospherical indicatrix of the Monge form germ $\left(\boldsymbol{x}_{f}, 0\right)$ is given as follows:

$$
\boldsymbol{x}_{f}^{-1}\left(H S\left(\boldsymbol{v}_{0}^{ \pm},-1\right)\right)=\left\{\left(u_{1}, \ldots, u_{n-1}\right) \mid \pm 2 f\left(u_{1}, \ldots, u_{n-1}\right)=u_{1}^{2}+\cdots+u_{n-1}^{2}\right\} .
$$

Since the height function of $x_{f}$ at $\boldsymbol{v}_{0}^{ \pm}$is

$$
h_{v_{0}^{ \pm}}(u)=-\sqrt{f^{2}\left(u_{1}, \ldots, u_{n-1}\right)+u_{1}^{2}+\cdots+u_{n-1}^{2}+1} \pm f\left(u_{1}, \ldots, u_{n-1}\right)+1,
$$

we can calculate the Hessian matrix, then we have Hess $\left(h_{v_{0}^{ \pm}}(0)\right)=\operatorname{Hess}( \pm f(0))$. Thus we conclude that $\mathrm{H}$-corank ${ }^{ \pm}\left(\boldsymbol{x}_{f}, 0\right)=(n-1)-\operatorname{rank}$ Hess $( \pm f(0))$.

On the other hand, since $f(0)=f_{u_{i}}(0)=0$, we may write

$$
f\left(u_{1}, \ldots, u_{n-1}\right)=\frac{\kappa_{1}}{2} u_{1}^{2}+\cdots+\frac{\kappa_{n-1}}{2} u_{n-1}^{2}+g\left(u_{1}, \ldots, u_{n-1}\right),
$$

where $g \in \mathfrak{M}_{n-1}^{3}$. Here, $\kappa_{1}, \ldots, \kappa_{n-1}$ are eigenvalues of $\left(f_{u_{i} u_{j}}(0)\right)$. Under this representation, we can easily calculate that $\boldsymbol{x}_{f, u_{i} u_{j}}(0)=\left(\delta_{i j}, f_{u_{i} u_{j}}(0), 0, \ldots, 0\right)$. It follows from this fact that

$$
\bar{h}_{i j}^{ \pm}(0)=-\delta_{i j} \pm f_{u_{i} u_{j}}(0)=\delta_{i j}\left(-1 \pm \kappa_{i}\right) .
$$

and $g_{i j}(0)=\delta_{i j}$. Therefore, we have $\bar{\kappa}_{i}^{ \pm}(0)=-1 \pm \kappa_{i}$ and

$$
K_{h}^{ \pm}(0)=\prod_{i=1}^{n-1} \bar{\kappa}_{i}^{ \pm}(0)=\prod_{i=1}^{n-1}\left(-1 \pm \kappa_{i}\right)
$$

The tangent hyperhorospherical indicatrix is given by

$$
\boldsymbol{x}_{f}^{-1}\left(H S\left(\boldsymbol{v}_{0},-1\right)\right)=\left\{\left(u_{1}, \ldots, u_{n-1}\right) \mid \bar{\kappa}_{1}^{ \pm}(0) u_{1}^{2}+\cdots+\bar{\kappa}_{n-1}^{ \pm}(0) u_{n-1}^{2} \pm 2 g\left(u_{1}, \ldots, u_{n-1}\right)=0\right\} .
$$




\section{Surfaces in Hyperbolic 3-space}

In this section we stick to the case when $n=3$. In this case we call $\boldsymbol{x}: U \longrightarrow H_{+}^{3}(-1)$ a surface, $H S\left(\boldsymbol{v}_{0},-1\right)$ a horosphere etc. By Proposition 8.2 and the classification of function germs [1], we have the following theorem.

Theorem 10.1 There exists an open dense subset $\mathcal{O} \subset \operatorname{Emb}\left(U, H_{+}^{3}(-1)\right)$ such that for any $\boldsymbol{x} \in \mathcal{O}$, the following conditions hold:

(1) The $H^{ \pm}$-parabolic set $K_{h}^{-1}(0)$ is a regular curve. We call such a curve the $H^{ \pm}$-parabolic curve.

(2) The hyperbolic Gauss indicatrix $\mathbb{L}^{ \pm}$along the $H^{ \pm}$-parabolic curve is the cuspdialedge except isolated points. At these points $\mathbb{L}^{ \pm}$is the swallowtail.

Here, a map germ $f:\left(\mathbb{R}^{2}, \boldsymbol{a}\right) \longrightarrow\left(\mathbb{R}^{3}, \boldsymbol{b}\right)$ is called a cuspidaledge if it is $\mathcal{A}$-equivalent to the germ $\left(u_{1}, u_{2}^{2}, u_{2}^{3}\right)$ (cf., Fig. 1) and a swallowtail if it is $\mathcal{A}$-equivalent to the germ $\left(3 u_{1}^{4}+\right.$ $\left.u_{1}^{2} u_{2}, 4 u_{1}^{3}+2 u_{1} u_{2}, u_{2}\right)$ (cf., Fig.1).

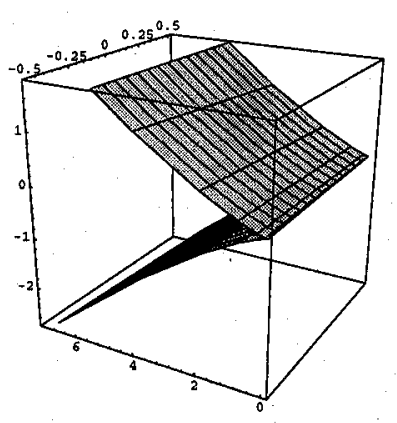

cuspidal edge

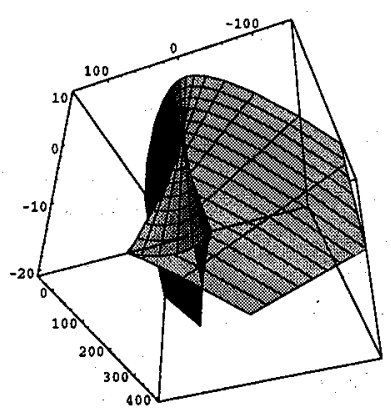

swallowtail

Fig. 1.

The assertion of Theorem 10.1 can be interpreted that the Legendrian lift $\mathcal{L}^{ \pm}$of the hyperbolic Gauss indicatrix $\mathbb{L}^{ \pm}$of $\boldsymbol{x} \in \mathcal{O}$ is Legendrian stable at each point. Since $\mathcal{L}^{ \pm}$is the Legendrian covering of the Lagrangian map $\widetilde{\mathcal{L}^{ \pm}}$associated to the hyperbolic Gauss map $\widetilde{\mathbb{L}^{ \pm}}$, it has been known that the corresponding singularities of $\widetilde{\mathbb{L}^{ \pm}}$are folds or cusps [1]. Hence, we have the following corollary.

Corollary 10.2 Let $\mathcal{O} \subset \operatorname{Emb}\left(U, H_{+}^{3}(-1)\right)$ be the same open dense subset as in Theorem 10.1. For any $\boldsymbol{x} \in \mathcal{O}$, the followings hold:

(1) $A H^{ \pm}$-parabolic point $u_{0} \in U$ is a fold of the hyperbolic Gauss map if and only if it is a cuspidal edge of the hyperbolic Gauss indicatrix.

(2) A $H^{ \pm}$-parabolic point $u_{0} \in U$ is a cusp of the hyperbolic Gauss map if and only if it is a swallowtail of the hyperbolic Gauss indicatrix.

Here, a map germ $f:\left(\mathbb{R}^{2}, \boldsymbol{a}\right) \longrightarrow\left(\mathbb{R}^{2}, \boldsymbol{b}\right)$ is called a fold if it is $\mathcal{A}$-equivalent to the germ $\left(u_{1}, u_{2}^{2}\right)$ and a cusp if it is $\mathcal{A}$-equivalent to the germ $\left(u_{1}, u_{2}^{3}+u_{1} u_{2}\right)$.

Following the terminology of Whitney[24], we say that a surface $\boldsymbol{x}: U \longrightarrow H_{+}^{3}(-1)$ has the excellent hyperbolic Gauss indicatrix $\mathbb{L}^{ \pm}$if $\mathcal{L}^{ \pm}$is a stable Legendrian immersion at each point. 
In this case, the hyperbolic Gauss indicatrix $\mathbb{L}^{ \pm}$has only cuspidaledges and swallowtails as singularities. Theorem 10.1 asserts that a surface with the excellent hyperbolic Gauss indicatrix is generic in the space of all surfaces in $H_{+}^{3}(-1)$. We now consider the geometric meanings of cuspidaledges and swallowtails of the hyperbolic Gauss indicatrix. We have the following results analogous to the results in Banchoff et al[2].

Theorem 10.3 Let $\mathbb{L}^{ \pm}:\left(U, u_{0}\right) \longrightarrow\left(H_{+}^{3}(-1), \boldsymbol{v}_{0}\right)$ be the excellent hyperbolic Gauss indicatrix of a surface $\boldsymbol{x}$ and $h_{v_{0}^{ \pm}}:\left(U, u_{0}\right) \longrightarrow \mathbb{R}$ be the horospherical height function germ at $\boldsymbol{v}_{0}^{ \pm}=\mathbb{L}^{ \pm}\left(u_{0}\right)$. Then we have the following:

(1) $u_{0}$ is an $H^{ \pm}$-parabolic point of $\boldsymbol{x}$ if and only if $\mathrm{H}$-corank ${ }^{ \pm}\left(\boldsymbol{x}, u_{0}\right)=1$ (i.e., $u_{0}$ is not a horospherical point of $\boldsymbol{x}$ ).

(2) If $u_{0}$ is an $H^{ \pm}$-parabolic point of $\boldsymbol{x}$, then $h_{v_{0}^{ \pm}}$has the $A_{k}$-type singularity for $k=2,3$.

(3) Suppose that $u_{0}$ is an $H^{ \pm}$-parabolic point of $\boldsymbol{x}$. Then the following conditions are equivalent:

(a) $\mathbb{L}^{ \pm}$has a cuspidaledge at $u_{0}$

(b) $h_{v_{0}^{ \pm}}$has the $A_{2}$-type singularity.

(c) ${\mathrm{H}-\mathrm{ord}^{ \pm}}^{ \pm}\left(\boldsymbol{x}, u_{0}\right)=2$.

(d) Tangent horospherical indicatrix is a ordinary cusp, where a curve $C \subset \mathbb{R}^{2}$ is called a ordinary cusp if it is diffeomorphic to the curve given by $\left\{\left(u_{1}, u_{2}\right) \mid u_{1}^{2}-u_{2}^{3}=0\right\}$.

(e) For each $\varepsilon>0$, there exist two distinct points $u_{1}, u_{2} \in U$ such that $\left|u_{0}-u_{i}\right|<\varepsilon$ for $i=1,2$, both of $u_{1}, u_{2}$ are not $H^{ \pm}$-parabolic points and the tangent horosphere to $M=\boldsymbol{x}(U)$ at $u_{1}, u_{2}$ are parallel.

(4) Suppose that $u_{0}$ is an $H^{ \pm}$-parabolic point of $\boldsymbol{x}$. Then the following conditions are equivalent:

(a) $\mathbb{L}^{ \pm}$has a swallowtail at $u_{0}$

(b) $h_{v_{0}^{ \pm}}$has the $A_{3}$-type singularity.

(c) $\mathrm{H}$-ord ${ }^{ \pm}\left(\boldsymbol{x}, u_{0}\right)=3$.

(d) Tangent horospherical indicatrix is a point or a tachnodal, where a curve $C \subset \mathbb{R}^{2}$ is called a tachnodal if it is diffeomorphic to the curve given by $\left\{\left(u_{1}, u_{2}\right) \mid u_{1}^{2}-u_{2}^{4}=0\right\}$.

(e) For each $\varepsilon>0$, there exist three distinct points $u_{1}, u_{2}, u_{3} \in U$ such that $\left|u_{0}-u_{i}\right|<\varepsilon$ for $i=1,2,3$ and the tangent horosphere to $M=\boldsymbol{x}(U)$ at $u_{1}, u_{2}, u_{3}$ are parallel.

(f) For each $\varepsilon>0$, there exist two distinct points $u_{1}, u_{2} \in U$ such that $\left|u_{0}-u_{i}\right|<\varepsilon$ for $i=1,2$ and the tangent horosphere to $M=\boldsymbol{x}(U)$ at $u_{1}, u_{2}$ are equal.

Proof. We have shown that $u_{0}$ is an $H^{ \pm}$-parabolic point if and only if $H$-corank ${ }^{ \pm}\left(\boldsymbol{x}, u_{0}\right) \geq 1$. Since $n=3$, we have $\mathrm{H}$-corank ${ }^{ \pm}\left(\boldsymbol{x}, u_{0}\right) \leq 2$. Since the horospherical height function germ $H:\left(U \times L C_{+}^{*},\left(u_{0}, \boldsymbol{v}_{0}\right)\right) \longrightarrow \mathbb{R}$ can be considered as a generating family of the Legendrian

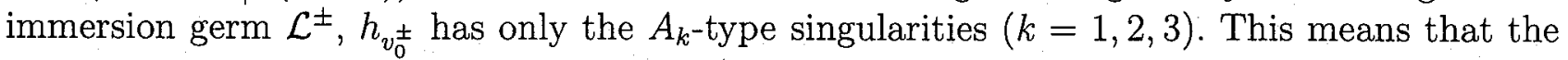
corank of the Hessian matrix of $h_{v_{0}^{ \pm}}$at an $H^{ \pm}$-parabolic point is 1 . The assertion (2) also follows. By the same reason, the conditions (3);(a),(b),(c) (respectively, (4); (a),(b),(c)) are equivalent. If the height function germ $h_{v_{0}^{ \pm}}$has the $A_{2}$-type singularity, it is $\mathcal{K}$-equivalent to the germ $\pm u_{1}^{2}+u_{2}^{3}$. Since the $\mathcal{K}$-equivalence send the zero level sets, the tangent horospherical indicatrix is diffeomorphic to the curve given by $=0$. This is the ordinary cusp. The normal form for the $A_{3}$-type singularity is given by $\pm u_{1}^{2}+u_{2}^{4}$, so that the tangent horospherical indicatrix is diffeomorphic to the curve $\pm u_{1}^{2}+u_{2}^{4}=0$. This means that the condition (3),(d) (respectively, $(4),(d))$ is also equivalent to the other conditions.

Suppose that $u_{0}$ is an H-parabolic point, then the hyperbolic Gauss map has only folds or cusps. If the point $u_{0}$ is a fold point, there is a neighbourhood of $u_{0}$ on which the hyperbolic 
Gauss map is 2 to 1 except the H-parabolic curve (i.e, fold curve). By Lemma 7.2, the condition (3), (e) is satisfied. If the point $u_{0}$ is a cusp, the critical value set is an ordinary cusp. By the normal form, we can understand that the hyperbolic Gauss map is 3 to 1 inside region of the critical vale. Moreover, the point $u_{0}$ is in the closure of the region. This means that the condition (4),(e) holds. We can also observe that near by a cusp point, there are 2 to 1 points which approach to $u_{0}$. However, one of those points are always $H^{ \pm}$-parabolic points. Since other singularities do not appear for in this case, so that the condition (3),(e) (respectively, (4),(e)) characterises a fold (respectively, a cusp).

If we consider the hyperbolic Gauss indicatrix in stead of the hyperbolic Gauss map, the only singularities are cuspidaledges or swallowtails. For the swallowtail point $u_{0}$, there are self intersection curve (cf., Fig. 1) approaching to $u_{0}$. On this curve, there are two distinct point $u_{1}, u_{2}$ such that $\mathbb{L}^{ \pm}\left(u_{1}\right)=\mathbb{L}^{ \pm}\left(u_{2}\right)$. By Lemma 7.2 , this means that tangent horosphere to $M=\boldsymbol{x}(U)$ at $u_{1}, u_{2}$ are equal. Since there are no other singularities in this case, the condition (4),(f) characterise a swallowtail point of $\mathbb{L}^{ \pm}$. This completes the proof.

We can study more detailed properties of surfaces in Hyperbolic 3-space. Nevertheless, we stop here, these will be discussed in the forthcoming papers.

\section{Examples}

In the last part of the paper, we give some examples and draw their pictures. In order to draw pictures, we consider the Poincaré ball model. Let $D=\left\{\left(x_{1}, x_{2}, x_{3}\right) \mid x_{1}^{2}+x_{2}^{2}+x_{3}^{2}<1\right\}$ be the Poincaré ball model of Hyperbolic space. Here, we consider the hyperbolic metric

$$
d s^{2}=\frac{4\left(d x_{1}^{2}+d x_{2}^{2}+d x_{3}^{2}\right)}{1-x_{1}^{2}-x_{2}^{2}-x_{3}^{2}} .
$$

It has been known that there is the canonical isometric diffeomorphism $\Phi: H_{+}^{3}(-1) \longrightarrow D$ given by

For a H-Monge form

$$
\Phi\left(x_{0}, x_{1}, x_{2}, x_{3}\right)=\left(\frac{x_{1}}{x_{0}+1}, \frac{x_{2}}{x_{0}+1}, \frac{x_{3}}{x_{0}+1}\right) .
$$

$$
\boldsymbol{x}_{f}\left(u_{1}, u_{2}\right)=\left(\sqrt{f^{2}\left(u_{1}, u_{2}\right)+u_{1}^{2}+u_{2}^{2}+1}, f\left(u_{1}, u_{2}\right), u_{1}, u_{1}\right)
$$

the composition $\Phi \circ \boldsymbol{x}_{f}\left(u_{1}, u_{2}\right)$ is given by

$$
\left(\frac{f\left(u_{1}, u_{2}\right)}{\sqrt{f^{2}\left(u_{1}, u_{2}\right)+u_{1}^{2}+u_{2}^{2}+1}}, \frac{u_{1}}{\sqrt{f^{2}\left(u_{1}, u_{2}\right)+u_{1}^{2}+u_{2}^{2}+1}}, \frac{u_{2}}{\sqrt{f^{2}\left(u_{1}, u_{2}\right)+u_{1}^{2}+u_{2}^{2}+1}}\right) .
$$

Therefore, for any function $f\left(u_{1}, u_{2}\right)$ with $f(0)=f_{u_{i}}(0)=0$, we can draw the picture of the surface in $D$. For the horosphere

$$
\boldsymbol{h}^{ \pm}\left(u_{1}, u_{2}\right)=\left(\frac{u_{1}^{2}+u_{2}^{2}+2}{2}, \pm \frac{u_{1}^{2}+u_{2}^{2}}{2}, u_{1}, u_{2}\right)
$$

we have

$$
\Phi \circ \boldsymbol{h}^{ \pm}\left(u_{1}, u_{2}\right)=\left( \pm \frac{u_{1}^{2}+u_{2}^{2}}{u_{1}^{2}+u_{2}^{2}+4}, \frac{2 u_{1}}{u_{1}^{2}+u_{2}^{2}+4}, \frac{2 u_{2}}{u_{1}^{2}+u_{2}^{2}+4}\right) .
$$

The picture of the horosphere $\Phi \circ \boldsymbol{h}^{+}(U)$ is given by the left side picture in Fig.2. 
Example 11.1 If $f\left(u_{1}, u_{2}\right)=\frac{1}{3} u_{1}^{3}+\frac{1}{2} u_{1}^{2}$ then $\kappa_{1}=1$ and $\kappa_{2}=0$. Then we have $\bar{\kappa}_{1}^{-}(0)=-1$ and $\bar{\kappa}_{2}^{-}(0)=-2$, so that the origin is not a $H^{-}$-parabolic point. However, $\bar{\kappa}_{1}^{+}(0)=-1$ and $\bar{\kappa}_{2}^{+}(0)=0$, then the origin is a $\mathrm{H}^{+}$-parabolic point. The positive tangent horospherical indicatrix is the ordinary cusp $u_{2}^{2}=\frac{2}{3} u_{1}^{3}$. Therefore, the hyperbolic Gauss indicatrix $\mathbb{L}^{-}$is non-singular at the origin and $\mathbb{L}^{+}$is a cuspidaledge at the origin. The surface and the intersection of the positive horosphere are depicted in Fig.2.
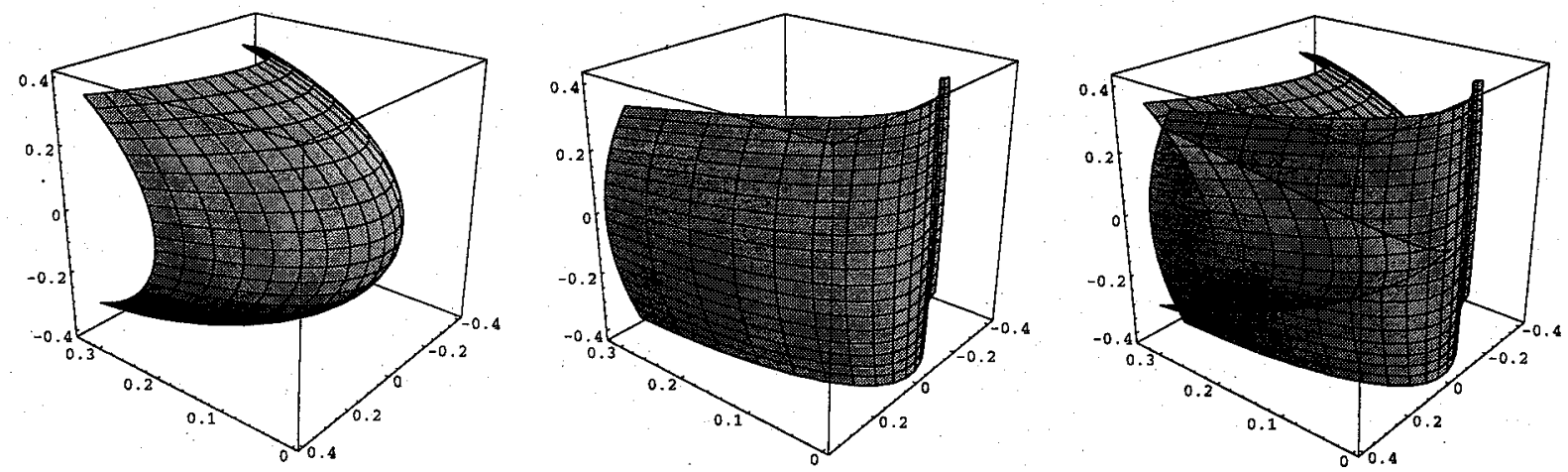

Fig. 2.

Example 11.2 Consider a function $f\left(u_{1}, u_{2}\right)=\frac{1}{2}\left(u_{1}^{2}+u_{1}^{4}\right)$, then $\kappa_{1}=1$ and $\kappa_{2}=0$. By the same reason as the previous example the origin is not a $\mathrm{H}^{-}$-parabolic point but a $\mathrm{H}^{+}$-parabolic point. The positive tangent horospherical indicatrix is the tachnode $u_{2}^{2}=u_{1}^{4}$. Therefore, the hyperbolic Gauss indicatrix $\mathbb{L}^{-}$is non-singular at the origin and $\mathbb{L}^{+}$is a swallowtail at the origin. The surface and the intersection of the positive horosphere are also depicted in Fig.3.
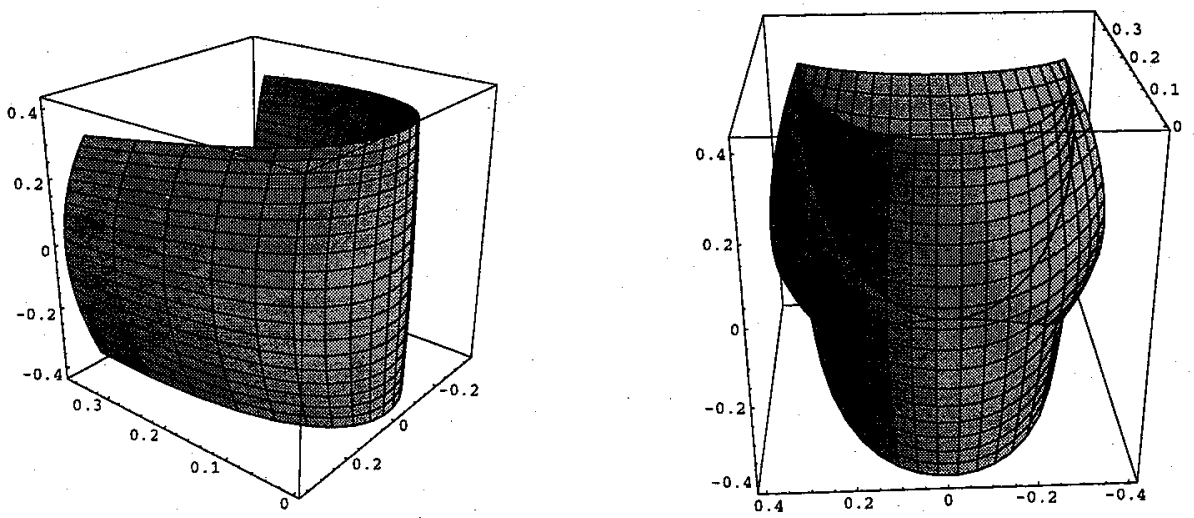

Fig. 3.

\section{References}

[1] V. I. Arnol'd, S. M. Gusein-Zade and A. N. Varchenko, Singularities of Differentiable Maps vol. I. Birkhäuser (1986)

[2] T. Banchoff, T. Gaffney and C. McCrory, Cusps of Gauss mappings. Research notes in Mathematics, Pitman, 55 (1982) 
[3] D. Bleeker and L. Wilson, Stability of Gauss maps. Illinois J. Math. 22, (1978), 279-289

[4] J. W. Bruce, The dual of generic hypersurfaces. Math. Scand., 49 (1981), 36-60

[5] J. W. Bruce and P. J. Giblin, Curves and singularities (second edition). Cambridge University press (1992)

[6] T. E. Cecil and P. J. Ryan, Distance functions and umbilic submanifolds of hyperbolic space. Nagoya Math. J., 74 (1979), 67-75

[7] C. L. Epstein The hyperbolic Gauss map and quasiconformal reflections. J. Reine Angew. Math., 372 (1986), 96-135

[8] M. Fukuda and T. Fukuda, Algebras $Q(f)$ determine the topological types of generic map germs. Invent. Math., 51 (1979), 231-237

[9] C. G. Gibson, K. Wirthmuller, A. A. du Plessis and E. J. Looijenga, Topological stability of smooth mappings. Lecture Notes in Math., 552 Springer-Verlag, (1976)

[10] S. Izumiya, On Legendrian singularities. Proceedings of AMS., 101 (1987), 748-752

[11] S. Izumiya, Topological properties of Legendrian singularities. Canadian Mathematical Bulletin, 34 (1991),357-363

[12] E. E. Landis, Tangential singularities. Funct. Anal. Appli., 15 (1981), 103-114

[13] J. Martinet, Singularities of Smooth Functions and Maps. London Math. Soc. Lecture Note Series, Cambridge Univ. Press, vol 58 (1982)

[14] J. N. Mather, Stability of $C^{\infty}$-mappings IV:Classification of stable germs by $\mathbb{R}$ algebras. Publi. Math. I.H.E.S., 37 (1970), 223-248

[15] J. N. Mather, How to stratify mappings and jet spaces. Lecture Notes in Math. 553, SpringerVerlag (1976)

[16] C. McCrory, T. Shifrin, Cusps of the projective Gauss map. J of Differential Geometry, 19 (1984), $257-276$

[17] C. McCrory, T. Shifrin and R. Varley, The Gauss map of a generic hypersurface in $\mathbb{P}^{4}$. J of Differential Geometry, 30 (1989), 689-759

[18] J. A. Montaldi, On contact between submanifolds. Michigan Math. J., 33 (1986), 195-199

[19] J. A. Montaldi, On generic composites of maps. Bull. London Math. Soc., 23 (1991), 81-85

[20] M. C. Romero Fuster, Sphere stratifications and the Gauss map. Proceedings of the Royal Soc. Edinburgh, 95A (1983), 115-136

[21] O. A. Platonova, Singularities in the problem of the quickest way round an obstrract. Funct. Anal. Appli.,15 (1981), 147-148

[22] Y.-H. Wan, Generic deformations of varieties. Trans. AMS., 259 (1980), 107-119

[23] G. Wassermann, Stability of Caustics. Math. Ann., 210 (1975), 43-50 
[24] H. Whitney, On singularities of mappings of Euclidean spaces I. Ann. of Math. 62 (1955), 374-410

[25] V. M. Zakalyukin, Lagrangian and Legendrian singularities. Funct. Anal. Appl., 10 (1976), 23-31

[26] V. M. Zakalyukin, Reconstructions of fronts and caustics depending one parameter and versality of mappings. J. Sov. Math., 27 (1984), 2713-2735 